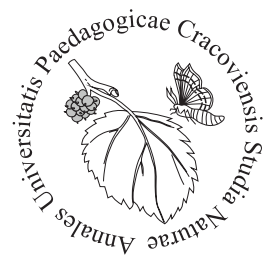

Krystyna Towpasz

Institute of Botany, Jagiellonian University, Kopernika 31 St., 31-501 Kraków, Poland; krystyna.towpasz@uj.edu.pl

\title{
Vascular plants of Pilzno surroundings (South-Eastern Poland)
}

Introduction

An extensive monographic study on the flora and geobotanical relations of the Strzyżów Foothills was created at the end of the last century (Towpasz, 1987, 1990). The flora of the Ciężkowice Foothills was analysed in a similar period (Kornaś et al., 1996). Later, only a few petty floristic publications were created to complement the flora of the Strzyżów Foothills (Oklejewicz et al., 2004; Wójcik, 2011; Towpasz, 2013). The natural environment of Pilsen surroundings has been recently presented in a monographic study (Towpasz, 2018). The aim of this study is floristic valorisation of the southern part of the Pilzno commune (South-Eastern Poland).

\section{General characteristics of the study area vegetation}

The study area belongs to the Western Outer Carpathians and is located within the macroregion of the Central Beskids Foothills and two mesoregions: Ciężkowice Foothills (Pogórze Ciężkowickie) and Strzyżów Foothills (Pogórze Strzyżowskie) (Fig. 1). The landscape is dominated by arable fields, locally meadows and pastures (Appendix $1 \mathrm{~A}-\mathrm{B})$. Forests occupy a small area and are preserved in places unfavorable to agriculture. Rare protected plants grow within them, among others: Matteucia struthiopteris, Staphyllea pinnata (Appendix 1C-D), or Scilla bifolia.

Meadows, arised and maintained on this area as a consequence of constant human intervention, such as: mowing, grazing, fertilisation. Depending on the degree of soil moisture, the following types of meadows are distinguished in this area: constantly and periodically moist and fresh meadows. On this area, pastures occupy a smaller area compared to meadows. For example, the association of water lilies is very rare in local ponds and oxbow lakes. Xerothermic vegetation develops here on dry, sunny slopes of hills and river valleys, mainly at southern exposure. Synanthropic communi- 


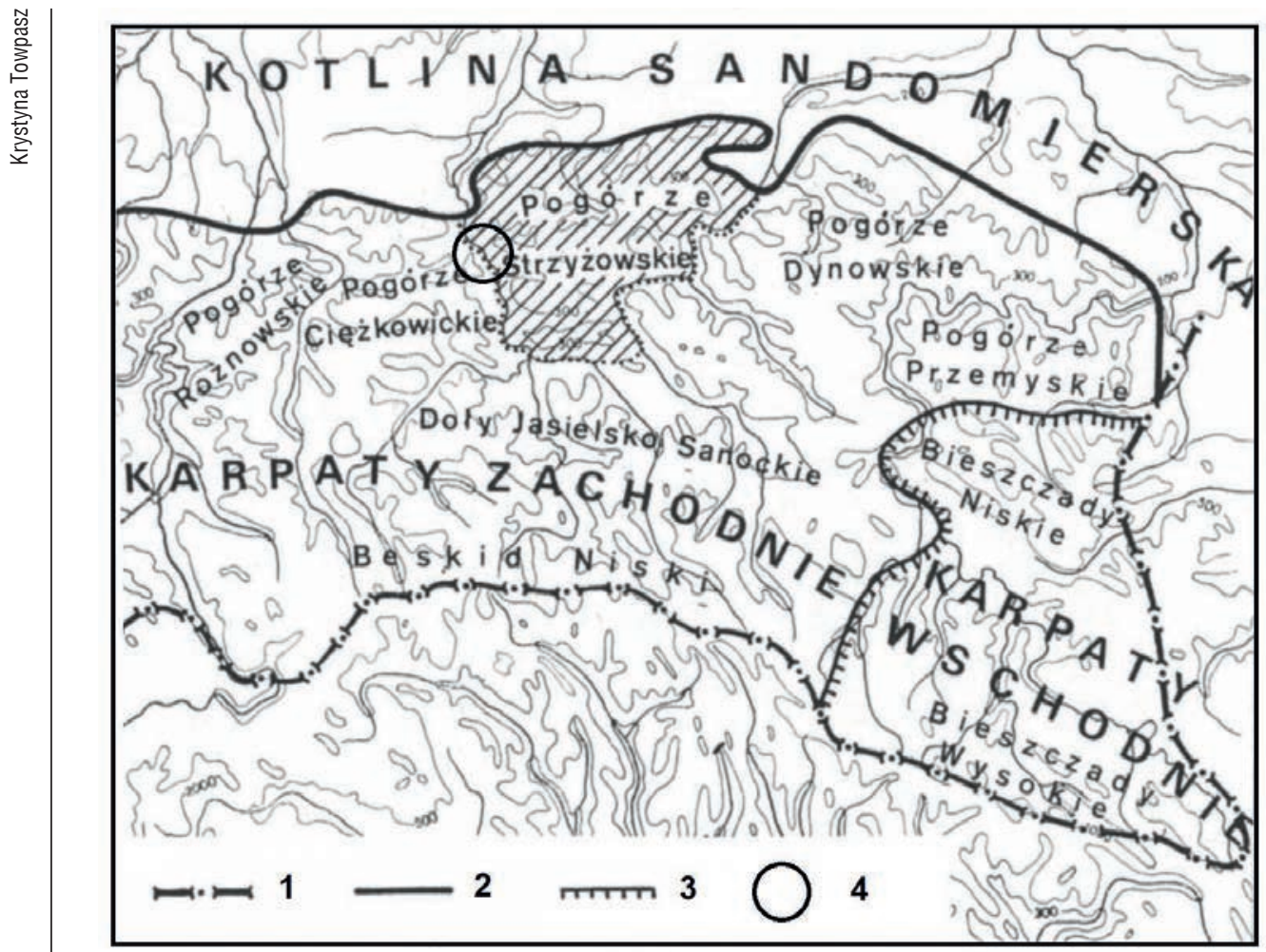

Fig. 1. Localisation of the study area against the regional divisionof the Eastern part of the Polish Carpathians (according Kondracki, 1978): 1 - state boundary, 2 - the northern limits of the Carpathians, 3 - boundary between the Western ana Eastern Carpathians, 4 - study region

ties, related to human activity, are creating among other as segetal vegetation in fields and gardens, and as ruderal vegetation accompanying human settlements, communication lines or industrial centers.

Research methods

A detailed monographic studies of the vascular plant flora of the Strzyżów and Ciężkowice Foothills dates from the end of the 20th century (Towpasz, 1987, 1990; Kornaś et al., 1997). In connection with the elaboration of a chapter on the natural environment of Pilzno (Towpasz, 2018), some of the materials contained in these studies related to the occurrence of vascular plants in the Pilzno commune were used, after their updating and verification in the field in recent years. To complement the flora of the Strzyżów Foothills, earlier studies were also used (Oklejewicz et al., 2004; Wójcik, 2011; Towpasz, 2013). 


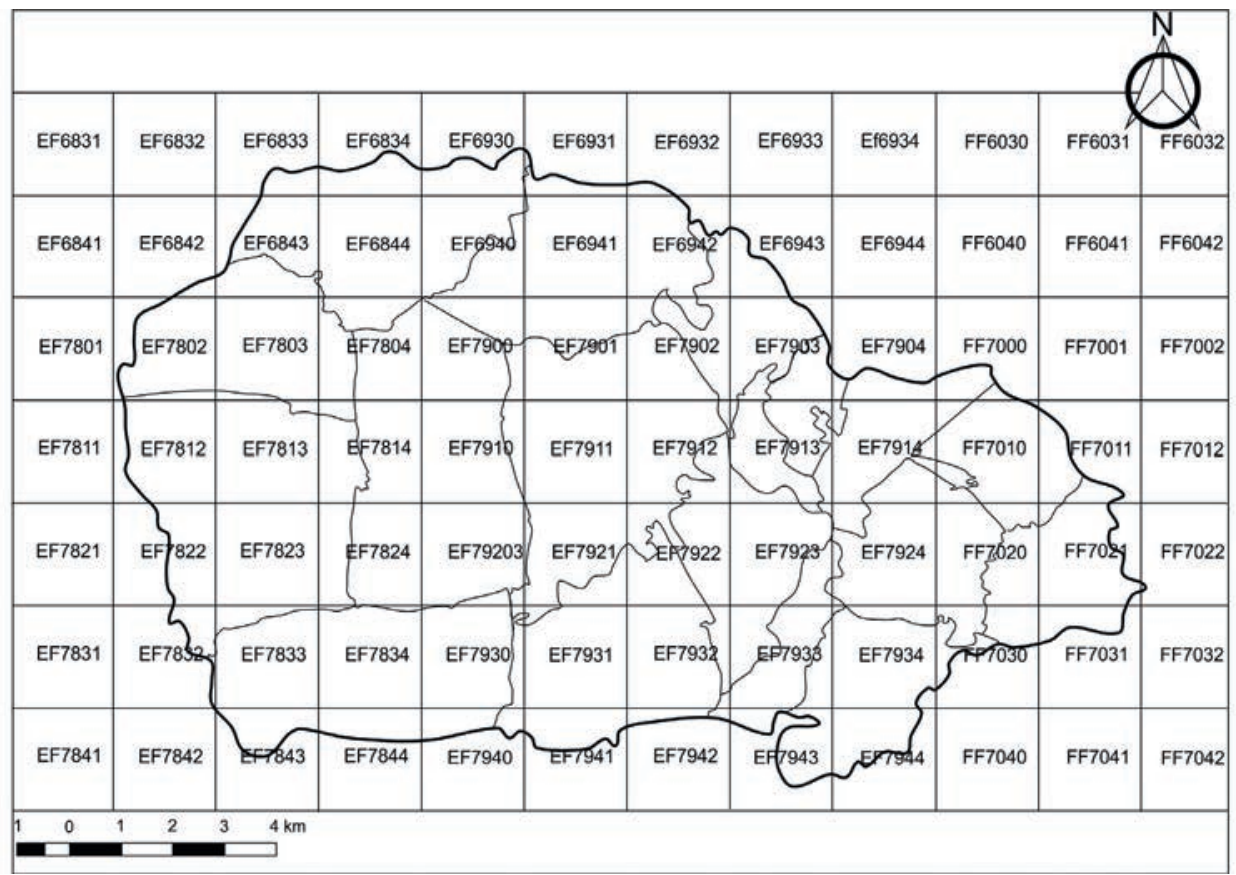

Fig. 2. Distribution of ATPOL grid squares in the area of Pilzno commune

The order of the species is given in alphabetical sequence, and their nomenclature is consistent with Mirek et al. (2002). All localities listed here were located in the ATPOL - the Polish geobotanical grid, $2 \mathrm{~km}$ long, in accordance with the methodology proposed by Zając (1978). Distribution of ATPOL grid squares in the area of Pilzno commune is shown in figure 2. Alien origin plants, mountain species (Pawłowski, 1925, 1972) and protected plants (Regulation of the Minister of the Environment of October 9 (...), 2014) are indicated in the list. Classification of alien species was adopted according to the atlas Zając and Zając (2001) and supplemented based on the study by Tokarska-Guzik et al. (2012). For alien species, found in semi-natural and natural habitats (agriophytes), their belonging to the group of invasive plants in the studied area is given in accordance with the work (Zając, Zając, 2015).

In the prepared floristic list, the following marks and abbreviations have been used: * - alien species, Grn. - Górne, Dln - Dolne, CF - Ciężkowice Foothills, SF - Strzyżów Foothills.

\section{Glossary of geographical names in the Pilzno commune}

The list includes geographical coordinates for the villages and signatures of the ATPOL grid squares. 
Bielowy $49^{\circ} 56^{\prime} 46^{\prime \prime} \mathrm{N} 21^{\circ} 19^{\prime} 36^{\prime \prime} \mathrm{E}$ (EF 7933 ) - village, $6 \mathrm{~km}$ SE from Pilzno (CF)

Dęborzyn $49^{\circ} 55^{\prime} 07^{\prime \prime} \mathrm{N} 21^{\circ} 18^{\prime} 59^{\prime \prime} \mathrm{E}$ (EF 7944 ) - village, about $7 \mathrm{~km}$ SE from Pilzno (CF)

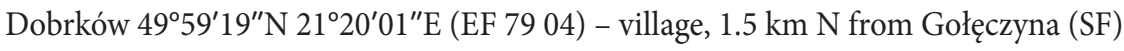

Dulczówka 49 $58^{\prime} 43^{\prime \prime} \mathrm{N} 21^{\circ} 17^{\prime} 29^{\prime \prime} \mathrm{E}$ (EF 79 12) - village, $1 \mathrm{~km}$ W from Pilzno (CF)

Gołęczyna $49^{\circ} 58^{\prime} 38^{\prime \prime} \mathrm{N} 21^{\circ} 20^{\prime} 36^{\prime \prime} \mathrm{E}$ (EF 79 14) - village, about $3 \mathrm{~km}$ SE from Pilzno (SF)

Jaworze Dolne $49^{\circ} 57^{\prime} 18^{\prime \prime} \mathrm{N} 21^{\circ} 21^{\prime} 08^{\prime \prime} \mathrm{E}$ (EF 7924 ) - village in the Wisłoka Valley, $8 \mathrm{~km}$ SE from Pilzno (SF)

Jaworze Górne 49 $55^{\prime} 59^{\prime \prime} \mathrm{N} 21^{\circ} 20^{\prime} 52^{\prime \prime} \mathrm{E}$ (EF 7934 ) - village, $2 \mathrm{~km}$ S from Jaworze Dolne (SF)

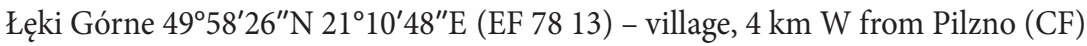

Mokrzec 49 $58^{\prime} 38^{\prime \prime} \mathrm{N} 21^{\circ} 19^{\prime} 36^{\prime \prime} \mathrm{E}$ (EF 79 13) - village, $1 \mathrm{~km}$ E from Gołęczyna (SF)

Parkosz $50^{\circ} 00^{\prime} 13^{\prime \prime} \mathrm{N} 21^{\circ} 18^{\prime} 50^{\prime \prime} \mathrm{E}$ (EF 6943 ) - village in the Wisłoka Valley, $3 \mathrm{~km}$ NE from Pilzno (SF)

Pilzno $49^{\circ} 58^{\prime} 43^{\prime \prime} \mathrm{N} 21^{\circ} 17^{\prime} 29^{\prime \prime} \mathrm{E}$ (EF 79 02; 79 03; 79 12) - city by the Tarnów - Dębica road, on the border of the Rożnów Foothills and the Strzyżów Foothills (CF, SF)

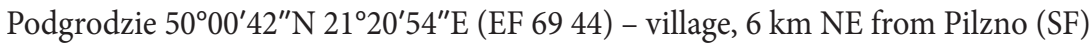

Rędziny $49^{\circ} 56^{\prime} 51^{\prime \prime} \mathrm{N} 21^{\circ} 18^{\prime} 08^{\prime \prime} \mathrm{E}$ (EF 7922 ) - hamlet of Strzegocice (CF)

Słotowa $49^{\circ} 56^{\prime} 43^{\prime \prime} \mathrm{N} 21^{\circ} 17^{\prime} 16^{\prime \prime} \mathrm{E}$ (EF 7931 ) - village, $2 \mathrm{~km}$ W from Strzegocice (CF)

Strzegocice $49^{\circ} 56^{\prime} 51^{\prime \prime} \mathrm{N} 21^{\circ} 18^{\prime} 08^{\prime \prime} \mathrm{E}$ (EF 7922 ) - village, $5 \mathrm{~km}$ S from Pilzno (CF)

Zagórze $49^{\circ} 55^{\prime} 19^{\prime \prime} \mathrm{N} 21^{\circ} 18^{\prime} 05^{\prime \prime} \mathrm{E}$ (EF 7933 ) - village, $1 \mathrm{~km}$ NW from Dęborzyn (CF)

Zwiernik $49^{\circ} 56^{\prime} 24^{\prime \prime} \mathrm{N} 21^{\circ} 12^{\prime} 34^{\prime \prime} \mathrm{E}$ (EF 7834 ) - village, $3 \mathrm{~km}$ S from Łęki Górne (CF)

\section{Results - list of species}

1. Abies alba Mill. - Higher montane zone species. In deciduous forests (oak-hornbeam forests, beech forests) and mixed forests: Podgrodzie (EF 69 44), Zwiernik (EF 78 34), Gołęczyna (EF 79 14), Jaworze Dln. (EF 79 24), Zagórze (EF 7933 ).

2. Acer campestre L. - In deciduous forests: Parkosz (EF 69 43), Podgrodzie (EF 69 44), Gołęczyna (EF 79 14).

3. A. platanoides L. - In deciduous forests: Parkosz (EF 69 43), Zwiernik (EF 78 34), Gołęczyna (EF 79 14).

4. A. pseudoplatanus L. - Higher montane zone species. In deciduous forests: Parkosz (EF 69 43), Łęki Grn. (EF 78 13).

5. Achillea millefolium L. - In the meadows: Parkosz (EF 69 43), Podgrodzie (EF 69 44), Łęki Grn. (EF 78 13), Pilzno (EF 79 12), Jaworze Dln. (EF 79 24).

6. Acinos arvensis (Lam.) Dandy - Balks and dry slopes: Parkosz (EF 69 43), Jaworze Dln. (EF 79 24).

7. Actaea spicata L. - Oak-hornbeam forest over the stream: Podgrodzie (EF 69 44).

8. Adoxa moschatellina L. - In forests: Parkosz (EF 69 43), Podgrodzie (EF 69 44). 
9. Aegopodium podagraria L. - Thickets over stream, roadsides: Parkosz (EF 69 43), Podgrodzie (EF 69 44), Łęki Grn. (EF 78 13), Pilzno (EF 79 12), Jaworze Dln. (EF 79 24).

10. ${ }^{\star}$ Aethusa cynapium L. subsp. agrestis (Wallr.) Dostál - Archaeophyte. Fields: Pilzno (EF 79 02).

11. Agrimonia eupatoria L. - Roadsides, balks: Parkosz (EF 69 43), Jaworze Dln. (EF 7924 ).

12. *Agrostemma githago L. - Archaeophyte. Fields: Bielowy (EF 7933 ).

13. Agrostis capillaris L. - In meadows and fields: Parkosz (EF 69 43), Podgrodzie (EF 6944 ), Zwiernik (EF 78 34), Pilzno (EF 79 02), Gołęczyna (EF 79 14), Bielowy (EF 7933 ).

14. A. gigantea Roth - Fields: Parkosz (EF 69 43), Pilzno (EF 79 12), Jaworze Dln. (EF 79 24), Słotowa (EF 79 31).

15. A. stolonifera L. - On stones of the Wisłoka River banks: Pilzno (EF 79 12), Jaworze Dln. (EF 79 24).

16. Ajuga reptans L. - In forests: Parkosz (EF 69 43), Podgrodzie (EF 69 44), Łęki Grn. (EF 78 13).

17. Alchemilla acutiloba Opiz - Pastures: Łęki Grn. (EF 78 13).

18. Alisma plantago-aquatica L. - In water reservoir and in drainage ditche: Parkosz (EF $6943)$.

19. Alliaria petiolata (M. Bieb.) Cavara \& Grande - In alluvial forests of the Wisłoka River: Parkosz (EF 69 43), Podgrodzie (EF 69 44), Łęki Grn. (EF 78 13), Pilzno (EF 79 02, EF 79 12), Jaworze Dln. (EF 79 24).

20. Allium oleraceum L. - Fields: Parkosz (EF 69 43), Podgrodzie (EF 69 44), Jaworze Dln. (EF 79 24).

21. A. scorodoprassum L. - On edges of thickets: Jaworze Dln. (EF 7924 ).

22. Alnus glutinosa (L.) Gaertn. - Oak-hornbeam forests: Parkosz (EF 69 43), Podgrodzie (EF 69 44), Łęki Grn. (EF 78 13), Jaworze Dln. (EF 79 24).

23. A. incana (L.) Moench - Higher montane zone species. In alluvial forests: Podgrodzie (EF 69 44).

24. Alopecurus geniculatus L. - Wet meadows: Pilzno (EF 79 02), Parkosz (EF 7903 ).

25. A. pratensis L. - Meadows: Parkosz (EF 69 43), Łęki Grn. (EF 78 13), Mokrzec (EF 79 13).

26. Alyssum alyssoides (L.) L. - On sandy fallow lands on the Wisłoka River: Parkosz (EF 69 43), Jaworze Dln. (EF 79 24).

27. *Amaranthus retroflexus L. - Epoecophyte. In fields, in root crops: Parkosz (EF 6943 ), Podgrodzie (EF 69 44), Gołęczyna (EF 79 14), Jaworze Dln. (EF 79 24).

28. *Anagallis arvensis L. - Archaeophyte. Fields: Parkosz (EF 69 43), Zwiernik (EF 78 34), Pilzno (EF 79 02), Mokrzec (EF 79 13).

29. ${ }^{\star}$ Anchusa officinalis L. - Archaeophyte. On fallow lands and on ruderal habitats: Podgrodzie (EF 69 44), Mokrzec (EF 79 13).

30. Anemone nemorosa L. - In deciduous forests: Podgrodzie (EF 69 44), Łęki Grn. (EF 78 13), Parkosz (EF 79 03). 
31. Angelica sylvestris L. - Meadows: Parkosz (EF 69 43), Podgrodzie (EF 69 44).

32. *Anthemis arvensis L. - Archaeophyte. Fields: Parkosz (EF 69 43), Podgrodzie (EF 69 44), Zwiernik (EF 78 34).

33. Anthriscus nitida (Wahlenb.) Hanzl. - Wet forest: Pilzno (EF 79 12).

34. A. sylvestris (L.) Hoffm. - In thickets of the Wisłoka River: Pilzno (EF 79 12), Strzegocice (EF 79 22).

35. Anthyllis vulneraria L. - In grasslands on slopes: Jaworze Dln. (EF 7924 ).

36. ${ }^{\star}$ Antoxanthum aristatum Boiss. - Holoagiophyte. On sandy dune in pine forest: Jaworze Dln. (EF 79 24).

37. A. odoratum L. - Meadows: Parkosz (EF 69 43), Podgrodzie (EF 69 44), Łęki Grn. (EF 78 13), Gołęczyna (EF 79 14).

38. *Apera spica-venti (L.) P. Beauv. - Archaeophyte. Fields: Parkosz (EF 69 43), Zwiernik (EF 78 34), Pilzno (EF 79 03).

39. *Aphanes arvensis L. - Archaeophyte. Fields: Parkosz (EF 69 43), Zwiernik (EF 78 34), Jaworze Dln. (EF 79 24).

40. Aposeris foetida (L.) Less. - Higher montane zone species. In deciduous forests: Bielowy (EF 79 33).

41. Arabidopsis thaliana (L.) Heynh. - On ruderal habitats: Podgrodzie (EF 69 44), Łęki Grn. (EF 78 13).

42. Arabis glabra (L.) Bernh. - On dry slopes: Parkosz (EF 69 43), Jaworze Dln. (EF 7924 ).

43. Arctium lappa L. - Roadsides: Parkosz (EF 69 43), Pilzno (EF 79 02).

44. A. minus (Hill) Bernh. - Roadside and alluvial forests of the Wisłoka River: Parkosz (EF 69 43), Dobrków (EF 79 04).

45. A. tomentosum Mill. - Roadside: Podgrodzie (EF 69 44).

46. Arenaria serpyllifolia L. - Roadsides: Podgrodzie (EF 69 44), Jaworze Dln. (EF 79 24).

47. *Armoracia rusticana P. Gaertn., B. Mey. \& Schreb. - Archaeophyte. Roadside: Pilzno (EF 7902 ).

48. Arrhenatherum elatius (L.) P. Beauv. ex J. Presl. \& C. Presl. - Meadows: Parkosz (EF 69 43), Podgrodzie (EF 69 44), Łęki Grn. (EF 78 13), Bielowy (EF 7933 ).

49. Artemisia campestris L. - Sand dunes and fallow land: Podgrodzie (EF 69 44), Gołęczyna (EF 79 14), Jaworze Dln.(EF 79 24).

50. A. vulgaris L. - Roadside: Parkosz (EF 69 43), Podgrodzie (EF 69 44), Łęki Grn. (EF 78 13), Pilzno (EF 79 12).

51. Asarum europaeum L. - Partially protected species. In deciduous forests: Parkosz (EF 69 43), Podgrodzie (EF 69 44), Jaworze Dln. (EF 79 24).

52. Asplenium ruta-muraria L. - On walls: Pilzno (EF 79 12), Strzegocice (EF 79 22).

53. A. trichomanes L. - In oak-hornbeam thickets: Jaworze Dln. (EF 7924 ).

54. ${ }^{\star}$ Aster novi-belgii L. - Hemiagriophyte. Invasive species. Roadside: Podgrodzie (EF 69 44). 
55. Astragalus glycyphyllos L. - On balks and roadsides: Zwiernik (EF 78 34), Jaworze Dln. (EF 79 24).

56. Athyrium filix-femina (L.) Roth - In forests: Parkosz (EF 69 43), Podgrodzie (EF 69 44), Łęki Grn. (EF 78 13), Jaworze Dln. (EF 79 24).

57. ${ }^{\star}$ Atriplex patula L. - Archaeophyte. Fields: Parkosz (EF 69 43), Zwiernik (EF 78 34).

58. ${ }^{\star}$ Avena fatua L. - Archaeophyte. Fields: Podgrodzie (EF 69 44), Jaworze Dln. (EF 7924 ).

59. ${ }^{\star B a l l o t a}$ nigra L. - Archaeophyte. Roadsides: Parkosz (EF 69 43), Podgrodzie (EF 69 44), Pilzno (EF 79 12).

60. Barbaraea vulgaris R. Br. - Roadside and meadow: Parkosz (EF 69 43).

61. Batrachium aquatile (L.) Dumort. - In water reservoir: Jaworze Dln. (EF 79 24).

62. Bellis perennis L. - Meadows: Parkosz (EF 69 43), Podgrodzie (EF 69 44), Łęki Grn. (EF 78 13).

63. Berteroa incana (L.) DC. - Roadsides: Parkosz (EF 69 43), Podgrodzie (EF 69 44).

64. Berula erecta (Huds.) Conville - In drainage ditches: Jaworze Dln. (EF 79 24).

65. Betula obscura Kotula - In forests: Parkosz (EF 69 43), Dobrków (EF 79 04).

66. B. pendula Roth - In forests: Podgrodzie (EF 69 44), Łęki Grn. (EF 78 13), Gołęczyna (EF 79 14).

67. ${ }^{\star}$ Bidens frondosa L. - Hemiagriophyte. Invasive species. On banks of drainage ditches: Dobrków (EF 79 04), Pilzno (EF 79 12).

68. B. tripartita L. - On banks of rivers and streams: Parkosz (EF 69 43), Zwiernik (78 34), Pilzno (EF 79 12).

69. Brachypodium sylvaticum (Huds.) P. Beauv. - In forests: Parkosz (EF 69 43), Podgrodzie (EF 69 44), Zwiernik (EF 78 34), Gołęczyna (EF 79 14), Jaworze Dln. (EF 79 24).

70. ${ }^{\star}$ Brassica nigra (L.) W. D. J. Koch - Epoecophyte. Roadside: Pilzno (EF 7902 ).

71. Briza media L. - Meadows: Słotowa (EF 79 31), Jaworze Dln. (EF 79 24).

72. ${ }^{\star}$ Bromus carinatus Hook. \& Arn. - Epoecophyte. On lawns and roadsides: Pilzno (EF 79 12), Jaworze Grn. (EF 79 34).

73. B. hordeaceus L. - Meadows: Parkosz (EF 69 43), Łęki Górne (EF 78 13), Pilzno (EF 79 12), Jaworze Dln. (EF 79 24).

74. B. inermis Leyss. - On dry slopes and roadsides: Parkosz (EF 69 43), Podgrodzie (EF 69 44), Dobrków (EF 79 04), Pilzno (EF 79 12).

75. ${ }^{\star}$ Bunias orientalis L. - Epoecophyte. On ruderal habitats: Jaworze Dln. (EF 79 24).

76. Calamagrostis arundinacea (L.) Roth - In pine forests: Jaworze Dln. (EF 79 24).

77. C. epigejos (L.) Roth - On balks, in thinned forests and on their outskirts: Podgrodzie (EF 69 44), Słotowa (EF 79 11), Gołęczyna (EF 79 14), Jaworze Dln. (EF 79 24).

78. Calluna vulgaris (L.) Hull - On edges of forests: Parkosz (EF 69 43), Gołęczyna (EF 79 14), Dobrków (EF 79 04), Jaworze Dln. (EF 79 24).

79. Caltha palustris L. - Wet meadows, banks of streams: Parkosz (EF 69 43), Jaworze Dln. (EF 79 24), Podgrodzie (EF 69 44). 
80. Calystegia sepium (L.) R. Br. - In alluvial forests of the Wisłoka River: Parkosz (EF 69 43), Podgrodzie (EF 69 44), Pilzno (EF 79 12).

81. Camelina microcarpa Andrz. - On sandy fallow lands: Jaworze Dln. (EF 79 24).

82. Campanula glomerata L. - Dry slopes: Jaworze Grn. (EF 79 34).

83. C. patula L. - Meadows: Podgrodzie (EF 69 44), Zwiernik (EF 78 34), Jaworze Dln. (EF 79 24).

84. C. persicifolia L. - In thickets of the Wisłoka River: Podgrodzie (EF 69 44).

85. C. rapunculoides L. - On cereal fields: Parkosz (EF 69 43), Pilzno (EF 79 02), Gołęczyna (EF 79 14).

86. C. trachelium L. - In thickets: Podgrodzie (EF 69 44), Gołęczyna (EF 79 14).

87. Capsella bursa pastoris (L.) Medik. - Roadsides: Podgrodzie (EF 69 34), Parkosz (EF 69 43), Łęki Grn. (EF 78 13), Pilzno (EF 79 02).

88. Cardamine amara L. - On the banks of streams: Parkosz (EF 69 43), Zwiernik (EF 78 34), Jaworze Dln. (EF 79 24).

89. C. impatiens L. - In deciduous forest: Jaworze Dln. (EF 79 24).

90. ${ }^{\star}$ Cardaria draba (L.) Desv. - Epoecophyte. Roadside: Mokrzec (EF 79 13).

91. ${ }^{\star}$ Carduus acanthoides L. - Archaeophyte. On balks and roadsides: Podgrodzie (EF 69 34), Parkosz (EF 69 43), Mokrzec (EF 79 13).

92. C. crispus L. - Fields: Pilzno EF 79 02), Dobrków (EF 79 04).

93. Carex brizoides L. - Oak-hornbeam forests: Parkosz (EF 69 43), Łęki Grn. (EF 78 13), Dobrków (EF 79 04), Jaworze Dln. (EF 79 24).

94. C. caryophyllea Latourr. - On lawn at Wisłoka's stone banks: Podgrodzie (EF 69 44).

95. C. cuprina (I. Sándor ex Heuff.) Nendtv. ex A. Kern. - Wet meadows: Parkosz (EF 69 43), Strzegocice (EF 79 22).

96. C. digitata L. - In forest: Podgrodzie (EF 69 44).

97. C. flacca Schreb. - Pasture: Jaworze Dln. (EF 79 24).

98. C. gracilis Curtis - On banks of drainage ditches: Parkosz (EF 69 43), Jaworze Dln. (EF 79 24).

99. C. hirta L. - Pastures: Parkosz (EF 69 43), Gołęczyna (EF 79 14).

100. C. nigra Reichard - Wet meadows: Łęki Grn. (EF 78 13), Strzegocice (EF 79 22).

101. C. ornithopoda Wild. - On stones of stream banks and on slope grassland: Parkosz (EF 69 43), Jaworze Dln. (EF 79 24).

102. C. ovalis Gooden. - Meadows: Podgrodzie (EF 69 44), Pilzno (EF 79 12).

103. C. pallescens L. - Meadow: Podgrodzie (EF 69 44).

104. C. panicea L. - Wet meadows: Łęki Grn. (EF 78 13), Jaworze Dln. (EF 79 24).

105. C. paniculata L. - Wet meadow: Jaworze Dln. (EF 79 24).

106. C. pendula Huds. - Lower montane zone species. In alluvial forest over stream: Podgrodzie (EF 69 44). 
107. C. pilosa Scop. - In forest: Parkosz (EF 69 43), Podgrodzie (EF 69 44), Jaworze Dln. (EF 79 24).

108. C. remota L. - Over stream: Dobrków (EF 7904 ).

109. C. spicata Huds. - On roadside embankment: Pilzno (EF 79 12).

110. C. sylvatica Huds. - Oak-hornbeam forests: Podgrodzie (EF 69 44), Zwiernik (EF 78 34), Jaworze Dln. (EF 79 24).

111. C. vesicaria L. - Wet meadows: Parkosz (EF 69 43), Dobrków (EF 7904 ).

112. C. vulpina L. - Wet meadows: Parkosz (EF 69 43), Zwiernik (EF 78 13), Strzegocice (EF 79 22).

113. Carlina vulgaris L. - Dry meadow: Parkosz (EF 6943 ).

114. Carpinus betulus L. - In deciduous forests, mainly in oak-hornbeam forests: Parkosz (EF 69 43), Podgrodzie (EF 69 44), Łęki Grn. (EF 78 13), Słotowa (EF 79 11), Gołęczyna (EF 79 14), Jaworze Dln. (EF 79 24).

115. Carum carvi L. - On meadows and balks: Podgrodzie (EF 6944$)$, Zwiernik (EF 78 34), Słotowa (EF 79 11), Jaworze Dln. (EF 79 24).

116. ${ }^{\star}$ Centaurea cyanus L. - Archaeophyte. Fields: Parkosz (EF 6943$)$, Podgrodzie (EF 6944 ), Łęki Grn. (EF 78 13), Jaworze Dln. (EF 79 24).

117. C. jacea L. - Meadows: Parkosz (EF 69 43), Podgrodzie (EF 69 44), Pilzno (EF 79 12), Jaworze Dln. (EF 79 24).

118. C. scabiosa L. - Balks: Podgrodzie (EF 69 44), Słotowa (EF 79 12), Jaworze Dln. (EF 79 24).

119. Centaurium erythraea Rafn - Partially protected species. Dry meadows: Parkosz (EF 69 43), Jaworze Dln. (EF 79 24).

120. Cephalanthera longifolia (L.) Fritsch - Protected species. Oak-hornbeam forest: Dęborzyn (EF 79 44).

121. Cerastium arvense L. - Fields: Podgrodzie (EF 69 44), Łęki Grn. (EF 78 13), Jaworze Dln. (EF 7924 ).

122. C. glomeratum Thull. - Field: Strzegocice (EF 79 22).

123. C. holosteoides Fr. emend. Hyl. - Meadows: Parkosz (EF 69 43), Łęki Grn. (EF 78 13).

124. C. semidecandrum L. - On sandy dunes: Podgrodzie (EF 69 44), Jaworze Dln. (EF 7924 ).

125. C. sylvaticum Waldst. \& Kit. - Lower montane zone species. Oak-hornbeam forests: Podgrodzie (EF 69 44), Jaworze Dln. (EF 79 24).

126. Cerasus avium (L.) Moench - Oak-hornbeam forest: Parkosz (EF 69 43), Podgrodzie (EF 69 44), Jaworze Dln. (EF 79 24).

127. Ceratophyllum demersum L. - In water reservoir: Mokrzec (EF 79 13).

128. Cerinthe minor L. - On dry slopes: Jaworze Dln. (EF 79 24).

129. Chaenorrhinum minor (L.) Lange - Roadsides: Parkosz (EF 69 43), Podgrodzie (EF 69 44), Pilzno (EF 79 02: EF 79 12).

130. Chaerophyllum aromaticum L. - Thickets: Pilzno (EF 79 12), Jaworze Dln. (EF 7924 ). 
131. Ch. hirsutum L. - Wet forest: Jaworze Dln. (EF 79 24).

132. Chamaenerion angustifolium (L.) Scop. - Thickets edge: Zwiernik (EF 78 34).

133. ${ }^{\star}$ Chamomilla suaveolens (Pursh) Rydb. - Epoecophyte. Fields and roadsides: Podgrodzie (EF 69 44), Łęki Grn. (EF 78 13), Pilzno (EF 79 02), Parkosz (EF 79 03), Dobrków (EF 79 04), Słotowa (EF 79 31).

134. Chelidonium maius L. - Roadsides: Podgrodzie (EF 69 44), Łęki Grn. (EF 78 13), Pilzno (EF 79 03), Słotowa (EF 79 11), Gołęczyna (EF 79 14).

135. Chenopodium album L. - Fields: Parkosz (EF 69 43), Podgrodzie (EF 69 44), Zwiernik (EF 78 34), Pilzno (EF 79 02, EF 79 12), Jaworze Dln. (EF 79 24).

136. Ch. glaucum L. - Field: Podgrodzie (EF 69 44).

137. ${ }^{\star}$ Ch. hybridum L. - Archaeophyte. On ruderal habitats: Podgrodzie (EF 69 44), Parkosz (EF 69 43).

138. Ch. polyspermum L. - Fields: Podgrodzie (EF 69 44), Dobrków (EF 79 04), Mokrzec (EF 79 13).

139. ${ }^{\star}$ Ch. strictum Roth - Epoecophyte. On ruderal habitat: Jaworze Dln. (EF 79 24).

140. Chimaphila umbellata (L.) W. P. C. Barton - In pine forests: Jaworze Dln. (EF 79 24).

141. Chrysosplenium alternifolium L. - In deciduous forests: Zwiernik (EF 78 24), Jaworze Dln. (EF 79 24), Podgrodzie (EF 79 44).

142. ${ }^{\star}$ Cichorium intybus L. - Archaeophyte. Roadsides: Podgrodzie (EF 69 44), Parkosz (EF 69 43), Słotowa (EF 79 11), Jaworze Dln. (EF 79 24).

143. Circaea lutetiana L. - In forests: Parkosz (EF 69 43), Podgrodzie (EF 69 44), Jaworze Dln. (EF 79 24).

144. Cirsium arvense (L.) Scop. - Fields: Parkosz (EF 69 43), Pilzno (EF 79 02, EF 79 12), Gołęczyna (EF 79 14).

145. C. oleraceum (L.) Scop. - Wet meadow: Dobrków (EF 79 04).

146. C. palustre (L.) Scop. - In balk: Podgrodzie (EF 69 44).

147. C. rivulare (Jacq.) All. - Wet meadows: Łęki Grn. (EF 78 13), Parkosz (EF 69 43), Strzegocice (EF 79 22).

148. C. vulgare (Savi) Ten. - Roadsides and pastures: Parkosz (EF 69 43), Podgrodzie (EF 69 44), Zwiernik (EF 78 34), Gołęczyna (EF 79 14), Strzegocice (EF 79 22).

149. Clinopodium vulgare L. - On dry slopes and balks: Gołęczyna (EF 79 14), Jaworze Dln. (EF 79 24), Zagórze (EF 79 33) ), Parkosz (EF 79 43).

150. ${ }^{\star}$ Consolida regalis Gray - Archaeophyte. Fields: Jaworze Dln. (EF 79 24), Bielowy (EF 7933 ).

151. Convallaria majalis L. - In oak-hornbeam forests: Podgrodzie (EF 69 44), Łęki Grn. (EF 78 13), Gołęczyna (EF 79 14), Jaworze Dln. (EF 79 24).

152. Convolvulus arvensis L. - Roadsides: Parkosz (EF 69 43), Podgrodzie (EF 69 44), Zwiernik (EF 78 34), Pilzno (EF 79 02, EF 79 12). 
153. `Conyza canadensis (L.) Cronquist - Epoecophyte. Roadsides: Parkosz (EF 69 43), Podgrodzie (EF 69 44), Mokrzec (EF 79 14).

154. Cornus sanguinea L. - Thickets: Parkosz (EF 69 43), Podgrodzie (EF 69 44), Gołęczyna (EF 79 14), Jaworze Dln. (EF 79 24).

155. Coronilla varia L. - In balks and on dry slopes grasslands: Parkosz (EF 69 43), Podgrodzie (EF 69 44), Jaworze Dolne (EF 79 24).

156. Corydalis solida (L.) Clairv. - In forests: Parkosz (EF 69 43), Podgrodzie (EF 69 44), Jaworze Dln. (EF 79 24).

157. Corylus avellana L. - In forests: Parkosz (EF 69 43), Podgrodzie (EF 69 44), Łęki Grn. (EF 78 13), Gołęczyna (EF 79 14), Jaworze Dln. (EF 79 24).

158. Corynephorus canecens (L.) P. Beauv. - On roadside and on sandy fallow lands: Podgrodzie (EF 69 44), Gołęczyna (EF 79 14), Jaworze Dln. (EF 79 24).

159. Crataegus monogyna Jacq. - In thickets of balks: Parkosz (EF 69 43), Podgrodzie (EF 69 44), Dobrków (EF 79 04), Mokrzec (EF 79 13), Strzegocice (EF 7922 ).

160. C. rhipidophylla Gand. var. lindmanii (Hrabětová) K. I. Chr. - In thickets of balk: Jaworze Grn.(EF 79 34).

161. C. rhipidophylla Gand. var. rhipidophylla - Thickets over the Wisłoka River: Podgrodzie (EF 69 44).

162. C. subsphaericea Gand. - In thickets of balk: Jaworze Dln. (EF 79 24) (Oklejewicz $\mathrm{i}$ in. 2004).

163. Crepis biennis L. - Meadows: Parkosz (EF 69 43), Podgrodzie (EF 69 44), Jaworze Dln. (EF 79 24).

164. C. capillaris (L.) Wallr. - Meadow: Jaworze Dln. (EF 7924$).$

165. C. paludosa (L.) Moench - In meadow and riparian forest: Parkosz (EF 69 43), Zwiernik (EF 78 34), Dobrków (EF 79 04).

166. C. tectorum L. - On sandy fallow area: Jaworze Dln. (EF 7924 ).

167. Cruciata glabra (L.) Ehrend. - On balk: Jaworze Dln. (EF 79 24).

168. Cucubalus baccifer L. - In wicker over the Wisłoka River: Podgrodzie (EF 6944 ).

169. Cuscuta epithymum (L.) L. - On meadow plants: Strzegocice (EF 7923 ).

170. C. europaea L. - On meadow plants: Parkosz (EF 69 43).

171. Cynoglossum officinale L. - On gravels of the Wisłoka River: Parkosz (EF 69 43), Jaworze Dln. (EF 79 24).

172. Cynosurus cristatus L. - Meadows: Podgrodzie (EF 69 44), Dobrków (EF 79 04).

173. Cystopteris fragilis (L.) Bernh. - On a stone wall: Strzegocice (EF 7922 ).

174. Dactylis glomerata L. - Meadows: Parkosz (EF 69 43), Łęki Grn. (EF 78 13).

175. D. polygama Horv. - In meadow and oak-hornbeam forest: Parkosz (EF 69 43), Dobrków (EF 79 04).

176. Dactylorhiza majalis (Rchb.) P. F. Hunt \& Summerh. - Partially protected species. Wet meadows: Podgrodzie (EF 69 44), Łęki Grn. (EF 78 13), Strzegocice (EF 79 22), Jaworze Dln. (EF 7924$)$. 
177. Daphne mezereum L. - Partially protected species. In deciduous forests: Parkosz (EF 69 43), Dobrków (EF 79 04), Dęborzyn (EF 79 43).

178. ${ }^{\star}$ Datura stramonium L. - Epoecophyte. Field: Gołęczyna (EF 79 14).

179. Daucus carota L. - Meadows: Podgrodzie (EF 69 44), Dobrków (EF 79 04).

180. Dentaria glandulosa Waldst. \& Kit. - Higher montane zone species. Oak-hornbeam forests: Podgrodzie (EF 69 44), Jaworze Dln. (EF 79 24).

181. Deschampsia caespitosa (L.) P. Beauv. - Meadows: Parkosz (EF 69 43), Jaworze Dln. (EF 79 24), Zagórze (EF 79 33).

182. ${ }^{\star}$ Descurainia sophia (L.) Webb ex Prantl - Archaeophyte. On ruderal habitats: Parkosz (EF 69 43), Podgrodzie (EF 69 44), Pilzno (EF 79 02).

183. Dianthus deltoides L. - On sandy grasslands: Podgrodzie (EF 69 44), Gołęczyna (EF 79 14), Jaworze Dln. (EF 79 24).

184. ${ }^{\star}$ Digitaria ischaemum (Schreb). H. L. Mühl. - Archaeophyte. On fallow area: Jaworze Dln. (EF 7924$)$.

185. ${ }^{\star}$ D. sanguinalis (L.) Scop. - Archaeophyte. On ruderal habitat: Podgrodzie (EF 69 44).

186. ${ }^{\star}$ Diplotaxis muralis (L.) DC. - Epoecophyte. Roadside: Pilzno (EF 7902 ).

187. Dipsacus laciniatus L. - On stones of the Wisłoka River bank: Pilzno (EF 79 12), Strzegocice (EF 79 22).

188. D. sylvestris Huds. - On stones of the Wisłoka River bank: Podgrodzie (EF 69 44), Pilzno (EF 79 12), Mokrzec (EF 79 13).

189. Dryopteris carthusiana (Vill.) H. P. Fuchs - In forests: Podgrodzie (EF 69 44), Zwiernik (EF 78 34), Dęborzyn (EF 79 44), Gołęczyna (EF 79 14).

190. D. dilatata (Hoffm.) A. Gray - All-mountain species. In forests: Podgrodzie (EF 6944$)$, Zwiernik (EF 78 34).

191. D. filix-mas (L.) Schott - In forests: Podgrodzie (EF 69 44), Gołęczyna (EF 79 14), Jaworze Dln. (EF 79 24).

192. D. expansa (C. Presl) Fraser-Jenk. \& Jermy - In forest: Jaworze Dln. (EF 79 24).

193. ${ }^{\star}$ Echinochloa crus-galli (L.) P. Beauv. - Archaeophyte. Fields: Parkosz (EF 69 43), Podgrodzie (EF 69 44).

194. * Echinocystis lobata (F. Michx.) Torr. \& A. Gray - Holoagriophyte. Invasive species. It grows in alluvial forests: Mokrzec (EF 79 13).

195. Echium vulgare L. - Roadsides: Podgrodzie (EF 69 44), Zwiernik (EF 78 34), Dobrków (EF 79 04), Jaworze Dln. (EF 79 24).

196. Eleocharis palustris (L.) Roem. \& Schult. - On banks of ditches: Parkosz (EF 69 43), Łęki Grn. (EF 78 13), Jaworze Dln. (EF 79 24).

197. Elymus caninus (L.) L. - In thickets of rivers and streams: Podgrodzie (EF 69 44), Pilzno (EF 79 12).

198. E. repens (L.) Gould - In field communities and roadsides: Parkosz (EF 69 43), Podgrodzie (EF 69 44), Łęki Grn. (EF 78 13), Zwiernik (EF 78 34), Gołęczyna (EF 79 14). 
199. Epilobium hirsutum L. - On edge of ditch: Podgrodzie (EF 69 44).

200. E. parviflorum Schreb. - On banks of streams: Podgrodzie (EF 69 44). Gołęczyna (EF 79 14), Jaworze Dln. (EF 79 24).

201. E. roseum Schreb. - On stones of the Wisłoka River bank: Pilzno (EF 79 12).

202. Epipactis helleborine (L.) Crantz - Partially protected species. Oak-hornbeam forests: Jaworze Dln. (EF 79 24), Jaworze Grn. (EF 79 34), Dęborzyn (EF 89 04).

203. E. purpurata Sm. - Protected species. Oak-hornbeam forest: Dęborzyn (EF 7944 ).

204. Equisetum arvense L. - In forests, fields, meadows, roadsides: Parkosz (EF 69 43), Podgrodzie (EF 69 44), Łęki Grn. (EF 78 13), Pilzno (EF 79 02, EF 79 03), Jaworze Dln. (EF 79 24).

205. E. fluviatile L. - In reeds on water reservoirs banks: Parkosz (EF 69 43), Łęki Grn. (EF 78 13).

206. E. hyemale L. - Oak-hornbeam forest: Podgrodzie (EF 69 44).

207. E. palustre L. - On banks of ditches: Łęki Grn. (EF 78 13), Dobrków (EF 79 04).

208. E. pratense Ehrh. - Wet oak-hornbeam forest: Łęki Grn. (EF 78 13).

209. E. ramosissimum Desf. - On stones of river banks: Łęki Grn. (EF 78 13), Jaworze Dln. (EF 79 24).

210. E. sylvaticum L. - In pine forests: Jaworze Dln. (EF 79 24), Słotowa (EF 7931 ).

211. E. telmateia Ehrh. - Lower montane zone species. In wet forests and on banks of streams: Podgrodzie (EF 69 44), Łęki Grn. (EF 78 13), Jaworze Dln. (EF 79 24).

212. Erigeron acris L. - Roadsides: Parkosz (EF 69 43), Zagórze (EF 7933 ).

213. Eriophorum angustifolium Honck.- Wet meadows: Parkosz (EF 69 43), Dęborzyn (EF 79 44).

214. E. latifolium Hoppe - Wet meadow: Łęki Grn. (EF 78 13).

215. Erodium cicutarium (L.) L'Hér. - On ruderal habitats: Parkosz (EF 69 43), Podgrodzie (EF 69 44).

216. Erophila verna (L.) Chevall. - On grasslands and dry slopes, fields: Parkosz (EF 69 43), Gołęczyna (EF 79 14).

217. Erysimum cheiranthoides L. - In fields and ruderal habitats: Pilzno (EF 79 12), Gołęczyna (EF 79 14), Jaworze Dln. (EF 79 24).

218. Euonymus europaea L. - In forests: Zwiernik (EF 78 34), Parkosz (EF 69 43), Dobrków (EF 79 04), Jaworze Dln. (EF 79 24).

219. E. verrucosa Scop. - In forests: Parkosz (EF 69 43), Podgrodzie (EF 69 44), Łęki Dln. (EF 79 42), Jaworze Dln. (EF 79 24).

220. Eupatorium cannabinum L. - In thickets above streams: Podgrodzie (EF 69 44). Jaworze Dln. (EF 79 24).

221. Euphorbia amygdaloides L. - In deciduous forests: Parkosz (EF 69 43), Dobrków (EF 79 04), Jaworze Dln. (EF 79 24).

222. E. cyparissias L. - On balks, pastures and dry meadows: Parkosz (EF 69 43), Podgrodzie (EF 69 44), Łęki Grn. (EF 78 13), Gołęczyna (EF 79 14). 
223. E. dulcis L. - Oak-hornbeam forest: Zwiernik (EF 78 34).

224. E. esula L. - On dry meadows, balks and pastures: Podgrodzie (EF 69 44), Pilzno (EF 79 12), Jaworze Dln. (EF 79 24).

225. ${ }^{\star}$ E. helioscopia L. - Archaeophyte. Fields and roadsides: Podgrodzie (EF 69 44), Pilzno (EF 79 02), Mokrzec (EF 79 13).

226. E. serrulata Thull. - Higher montane zone species. In thickets and on stones of rivers: Parkosz (EF 69 43), Podgrodzie (EF 69 44), Dobrków (EF 79 04), Jaworze Dln. (EF 79 24).

227. Euphrasia rostkoviana Hayne - On balk: Dobrków (EF 79 04).

228. Fagus sylvatica L. - In deciduous forests: Podgrodzie (EF 69 44), Gołęczyna (EF 79 14), Jaworze Dln. (EF 79 24).

229. *Fallopia convolvulus (L.) Á. Löve - Archaeophyte. Fields: Parkosz (EF 69 43), Pilzno (EF 79 02), Dobrków (EF 79 04), Jaworze Grn. (EF 79 34).

230. F. dumetorum (L.) Holub. - In thickets on the Wisłoka River: Podgrodzie (EF 69 44), Jaworze Dln. (EF 79 24).

231. Festuca gigantea (L.) Vill. - In forests and thickets above streams: Parkosz (EF 69 43), Podgrodzie (EF 69 44), Zwiernik (EF 78 34), Jaworze Dln. (EF 79 24), Zagórze (EF 79 33).

232. F. ovina L. - Meadows: Parkosz (EF 69 43), Gołęczyna (EF 79 14), Jaworze Dln. (EF 79 24).

233. F. pratensis Huds. - Meadows: Strzegocice (EF 79 22), Jaworze Dln. (EF 79 24).

234. F. rubra L. - Meadows: Parkosz (EF 69 43), Słotowa (EF 7931 ), Jaworze Dln. (EF 7924 ).

235. Ficaria verna Huds. - In the forests, on roadsides: Parkosz (EF 69 43), Podgrodzie (EF 69 44), Łęki Grn. (EF 78 13), Pilzno (EF 79 03).

236. Filipendula ulmaria (L.) Maxim. - Wet meadows: Parkosz (EF 69 43), Dobrków (EF 79 04).

237. Fragaria vesca L. - In forests, thickets and dry meadows: Parkosz (EF 69 43), Podgrodzie (EF 69 44), Łęki Grn. (EF 78 13), Jaworze Dln. (EF 79 24), Bielowy (EF 7933 ).

238. Frangula alnus Mill. - Forests and thickets: Parkosz (EF 69 43), Podgrodzie (EF 69 44), Gołęczyna (EF 79 14).

239. Fraxinus excelsior L. - In moist and wet forests: Parkosz (EF 69 43), Dobrków (EF 79 04).

240. Gagea lutea (L.) Ker Gawł. - Forests and thickets: Parkosz (EF 69 43), Podgrodzie (EF 69 44), Gołęczyna (EF 79 14).

241. Galega officinalis L. - On edges of thickets and on meadows: Parkosz (EF 69 43), Podgrodzie (EF 69 44), Strzegocice (EF 79 22).

242. Galeobdolon luteum Huds. - In forests: Parkosz (EF 69 43), Podgrodzie (EF 69 44), Gołęczyna (EF 79 14), Jaworze Dln. (EF 79 24).

243. Galeopsis bifida Boenn. - In forests: Parkosz (EF 69 43), Słotowa (EF 79 31).

244. ${ }^{*}$ G. ladanum L. - Archaeophyte. Field: Parkosz (EF 69 43).

245. G. pubescens Besser - In forests and on ruderal habitats: Parkosz (EF 69 43), Podgrodzie (EF 69 44), Zwiernik (EF 78 34), Pilzno (EF 79 12), Jaworze Dln. (EF 79 24). 
246. G. speciosa Mill. - In forests and thickets: Podgrodzie (EF 69 44), Dobrków (EF 79 04). 247. G. tetrahit L. - In forests: Gołęczyna (EF 79 14), Jaworze Grn. (EF 79 34).

248. ${ }^{\star}$ Galinsoga ciliata (Raf.) S. F. Blake - Epoecophyte. In ruderal habitats and on fields: Parkosz (EF 69 43), Pilzno (EF 79 12).

249. ${ }^{\star}$ G. parviflora Cav. - Epoecophyte. In ruderal habitats and on fields: Parkosz (EF 69 43), Podgrodzie (EF 69 44), Pilzno (EF 79 12).

250. Galium aparine L. - In forestss, thickets and on fields: Parkosz (EF 69 43), Łęki Grn. (EF 78 13), Pilzno (EF 79 02, EF 79 12).

251. G. mollugo L. - On meadows and balks: Łęki Grn. (EF 78 13), Zagórze (EF 7943 ).

252. G. odoratum (L.) Scop. - In forests: Podgrodzie (EF 69 44), Jaworze Dln. (EF 79 24).

253. G. palustre L. - Banks of ponds and wet meadows: Parkosz (EF 69 43), Bielowy (EF 7933 ).

254. G. rivale (Sibth. \& Sm.) - On banks of ditches: Parkosz (EF 6943 ).

255. G. schultesii Vest - In forests and thickets: Parkosz (EF 69 43), Jaworze Dln. (EF 7924 ).

256. G. verum L. - Meadows and forests: Parkosz (EF 69 43), Podgrodzie (EF 69 44), Strzegocice (EF 7922 ).

257. Gentianella ciliata (L.) Borkh. - In grassland of dry meadow: Dobrków (EF 7904 ).

258. ${ }^{\star}$ Geranium dissectum L. - Archaeophyte. Fields: Podgrodzie (EF 69 44), Mokrzec (EF 79 13), Bielowy (EF 7933 ).

259. G. palustre L. - Wet meadows: Strzegocice (EF 79 22), Jaworze Dln. (EF 79 24).

260. G. phaeum L. - All-mountain species. In riparian forest over the Wisłoka River: Parkosz (EF 69 43).

261. G. pratense L. - Meadows: Podgrodzie (EF 69 44), Łęki Grn. (EF 78 11), Bielowy (EF 7933 ).

262. ${ }^{\star}$ G. pusillum Burm. F. ex L. - Archaeophyte. Fields: Podgrodzie (EF 69 44), Jaworze Dln. (EF 7924$)$.

263. G. robertianum L. - In forest: Jaworze Dln. (EF 79 24).

264. G. sanguineum L. - On edge of thickets: Jaworze Dln. (EF 7924 ).

265. Geum urbanum L. - Wet forests: Łęki Grn. (EF 78 13), Parkosz (EF 69 43), Gołęczyna (EF 79 14), Jaworze Dln. (EF 79 24).

266. Glechoma hederacea L. - Roadsides: Parkosz (EF 69 43), Podgrodzie (EF 69 44), Łęki Grn. (EF 78 13), Zwiernik (EF 78 34), Pilzno (EF 79 12), Jaworze Dln. (EF 79 24).

267. G. hirsuta Waldst. \& Kit. - In forests: Podgrodzie (EF 69 44), Jaworze Dln. (EF 7924 ).

268. Glyceria maxima (Hartm.) Holub. - On banks of pond and over the Wisłoka River:

Parkosz (EF 69 43). Podgrodzie (EF 69 44).

269. Gnaphalium uliginosum L. - Wet fields: Parkosz (EF 69 43), Jaworze Dln. (EF 79 24).

270. Gymnocarpium dryopteris (L.) Newman - In forest: Jaworze Dln. (EF 79 24).

271. Hedera helix L. - Protected species. In forests: Parkosz (EF 69 43), Podgrodzie (EF 69 44), Łęki Grn. (EF 78 13), Dobrków (EF 79 04). 
272. ${ }^{\star}$ Helianthus tuberosus L. - Holoagriophyte. Invasive species. In alluvial forests over the Wisłoka River: Pilzno (EF 79 12).

273. Heracleum sphondylium L. - Meadows and roadsides: Podgrodzie (EF 69 44), Pilzno (EF 79 03), Dobrków (EF 79 04).

274. Hieracium lachenalii C. C. Gmel. - In forests: Bielowy (EF 79 33), Jaworze Dln. (EF 79 24).

275. H. murorum L. - In forests: Zwiernik (EF 78 34), Gołęczyna (EF 79 14).

276. H. pilosella L. - Dry meadows and pastures: Podgrodzie (EF 69 44), Gołęczyna (EF 79 14), Jaworze Dln. (EF 79 24), Bielowy (EF 79 33).

277. H. piloselloides Vill. - Dry meadows: Strzegocice (EF 7922 ).

278. H. sabaudum L. - In forests: Parkosz (EF 69 43), Jaworze Dln. (EF 79 24).

279. H. umbellatum L. - In forests: Podgrodzie (EF 69 44), Jaworze Dln. (EF 79 24), Słotowa (EF 7931$)$.

280. Holcus lanatus L. - Meadows: Łęki Grn. (EF 78 13), Gołęczyna (EF 79 14), Jaworze Dln. (EF 79 24).

281. Holosteum umbellatum L. - In slope of grassland: Słotowa (EF 79 31).

282. ${ }^{\star}$ Hordeum murinum L. - Archaeophyte. Roadside: Jaworze Dln. (EF 79 24).

283. Humulus lupulus L. - In alluvial forests over rivers and streams: Podgrodzie (EF 69 44), Parkosz (EF 69 43), Jaworze Dln. (EF 79 24), Bielowy (EF 79 33).

284. Hypericum hirsutum L. - In forests: Podgrodzie (EF 69 44), Jaworze Dln. (EF 79 24).

285. H. humifusum L. - Pasture: Podgrodzie (EF 69 44).

286. H. maculatum Crantz - Wet meadow: Słotowa (EF 7931 ).

287. H. perforatum L. - Meadows and roadsides: Parkosz (EF 6943 ), Podgrodzie (EF 6944 ), Pilzno (EF 79 12), Gołęczyna (Ef 79 14).

288. H. tetrapterum Fr. - Wet meadows: Dobrków (EF 79 04), Jaworze Dln. (EF 79 24).

289. Hypochoeris radicata L. - Meadows and pastures: Podgrodzie (EF 69 44), Parkosz (EF 69 43), Gołęczyna (EF 79 14), Jaworze Dln. (EF 79 24), Słotowa (EF 7931 ).

290. *Impatiens glandulifera Royle - Holoagriophyte. Invasive species. In alluvial forest: Dobrków (EF 79 04).

291. I. noli-tangere L. - Wet forests: Parkosz (EF 69 43), Jaworze Dln. (EF 79 24).

292. ${ }^{\star}$ I. parviflora DC. - Holoagriophyte. Invasive species. In ruderal habitat: Pilzno (EF 79 12).

293. Inula britannica L. - Meadows: Parkosz (EF 69 43), Jaworze Dln. (EF 79 24).

294. ${ }^{\star}$ I. helenium L. - Hemiagriophyte. On edges of thickets: Bielowy (EF 7933 ).

295. Iris pseudacorus L. - On banks of ditches: Strzegocice (EF 7922 ).

296. Isopyrum thalictroides L. - In forest: Podgrodzie (EF 69 44).

297. Jasione motana L. - On sandy dunes and fallow lands: Podgrodzie (EF 69 44), Gołęczyna (EF 79 14), Jaworze Dln. (EF 79 24). 
298. *Juglans regia L. - Holoagriophyte. Currently in study area it settled on balks and in forests: Rędziny (EF 79 22), Jaworze Dln. (EF 79 24).

299. Juncus articulatus L. emend. K. Richt. - Meadows: Podgrodzie (EF 69 44), Pilzno (EF 79 12).

300. J. bufonius L. - In wet fields and ditches: Podgrodzie (EF 69 44), Dobrków (EF 79 04), Pilzno (EF 79 12).

301. J. conglomeratus L. emend. Leers - Meadows: Parkosz (EF 69 43), Jaworze Dln. (EF 79 24).

302. J. inflexus L. - Meadows: Parkosz (EF 69 43), Podgrodzie (EF 69 44), Jaworze Dln. (EF 7924$)$.

303. ${ }^{\star}$ J. tenuis Willd. - Hemiagriophyte. Invasive species. On roadsides and pastures: Podgrodzie (EF 69 44), Bielowy (EF 7933 ).

304. Juniperus communis L. - Thickets: Parkosz (EF 69 43), Jaworze Dln. (EF 79 24).

305. Knautia arvensis (L.) J. M. Coult. - Medow: Słotowa (EF 79 31).

306. ${ }^{\star}$ Lactuca serriola L. - Archaeophyte. Roadside: Podgrodzie (EF 69 44).

307. ${ }^{\star}$ Lamium album L. - Archaeophyte. Roadsides: Łęki Grn. (EF 78 13), Parkosz (EF 69 43), Pilzno (79 12), Jaworze Dln. (EF 79 24).

308. ${ }^{\star}$ L. amplexicaule L. - Archaeophyte. Fields: Jaworze Dln. (EF 79 24).

309. L. maculatum L. - On roadsides and ruderal habitats and in thickets above streams: Parkosz (EF 69 43), Podgrodzie (EF 69 44), Pilzno (EF 79 12), Zagórze (EF 7933 ).

310. ${ }^{\star}$ L. purpureum L. - Archaeophyte. Fields: Parkosz (EF 69 43), Podgrodzie (EF 69 44), Pilzno (EF 79 03), Jaworze Dln. (EF 79 24).

311. Lapsana communis L. - Fields and roadsides: Zwiernik (EF 78 34), Pilzno (79 12), Mokrzec (EF 79 13).

312. Lathyrus pratensis L. - Meadows: Rędziny (EF 79 22), Bielowy (EF 7933 ).

313. ${ }^{\star}$ L. tuberosus L. - Archaeophyte. On cereal fields: Parkosz (EF 69 43), Pilzno (EF 79 12), Gołęczyna (EF 79 14), Jaworze Dln. (EF 79 24), Bielowy (EF 7933 ).

314. L. vernus (L.) Bernh. - In forests: Podgrodzie (EF 69 44), Parkosz (EF 6943 Dobrków (EF 7904 ).

315. Lavathera thuringiaca L. - In thickets over the Wisłoka River: Podgrodzie (EF 69 44), Jaworze Dln. (EF 79 24).

316. Lembotropis nigricans (L.) Griseb. - On edge of pine forest: Gołęczyna (EF 79 14).

317. Lemna minor L. - In pond: Parkosz (EF 69 43), Jaworze Dln. (EF 79 24).

318. L. trisulca L. - In pond: Parkosz (EF 69 43).

319. Leontodon autumnalis L. - Meadows: Strzegocice (EF 7922 ).

320. L. hispidus L. - Roadsides and meadows: Podgrodzie (EF 69 44), Strzegocice (EF 7922 ), Jaworze Dln. (EF 79 24).

321. *Leonurus cardiaca L. - Archaeophyte. On ruderal habitats: Parkosz (EF 69 43), Podgrodzie (EF 69 44), Pilzno (EF 79 12), Jaworze Dln. (EF 79 24). 
322. ${ }^{\star}$ Lepidium campestre (L.) R. Br. - Archaeophyte. On ruderal habitat: Jaworze Dln. (EF 7924$)$.

323. ${ }^{\star}$ L. ruderale L. - Archaeophyte. On ruderal habitat: Pilzno (EF 79 12).

324. Leucanthemum vulgare Lam. - Meadows: Parkosz (EF 69 43), Gołęczyna (EF 79 14), Jaworze Dln. (EF 79 24).

325. Ligustrum vulgare L. - In xerothermic thickets in balks and in thickets over the Wisłoka River: Łęki Grn. (EF 78 13), Jaworze Dln. (EF 79 24).

326. Lilium martagon L. - Protected species. In oak-pine forest: Jaworze Dln. (EF 7924 ).

327. Linaria vulgaris Mill. - On balks: Podgrodzie (EF 69 44), Zwiernik (EF 78 34), Pilzno (EF 79 12), Bielowy (EF 79 33).

328. ${ }^{\star}$ Lithospermum arvense L. - Archaeophyte. Fields: Parkosz (EF 69 43), Gołęczyna (EF 79 14), Jaworze Dln. (EF 79 24).

329. ${ }^{*}$ Lolium multiflorum Lam. - Epoecophyte. In meadows and fields in cereal crops: Parkosz (EF 69 43), Pilzno (EF 79 02, EF 79 12), Bielowy (EF 7933 ).

330. L. perenne L. - Meadows and pastures: Podgrodzie (EF 69 44), Parkosz (EF 69 43), Pilzno (EF 79 12).

331. Lonicera xylosteum L. - In deciduous forests: Podgrodzie (EF 69 44), Jaworze Dln. (EF 79 24).

332. Lotus corniculatus L. - Meadows: Podgrodzie (EF 69 44), Parkosz (EF 69 43), Bielowy (EF 7933 ).

333. L. uliginosus Schkuhr. - Wet meadow: Parkosz (EF 69 43).

334. Luzula campestris (L.) DC. - Meadows: Podgrodzie (EF 69 44), Parkosz (EF 69 43), Łęki Grn. (EF 78 13), Słotowa (EF 79 31).

335. L. multiflora (Retz.) Lej. - Meadows: Gołęczyna (EF 79 14), Bielowy (EF 79 33).

336. L. pilosa (L.) Willd. - In forests: Parkosz (EF 69 43), Podgrodzie (EF 69 44), Gołęczyna (EF 79 14), Jaworze Dln. (EF 79 24), Słotowa (EF 79 31).

337. Lychnis flos-cuculi L. - Bank of pond: Parkosz (EF 69 43), Bielowy (EF 79 33).

338. ${ }^{\star}$ Lycium barbarum L. - Epoecophyte. Roadsides: Parkosz (EF 69 43), Pilzno (EF 79 12), Strzegocice (EF 79 22).

339. Lycopus europaeus L. - On banks of streams: Podgrodzie (EF 69 44), Zwiernik (EF 78 34), Dobrków (EF 79 04).

340. Lysimachia nemorum L. - Higher montane zone species. In forest: Jaworze Dln. (EF 79 24).

341. L. nummularia L. - Meadows: Parkosz (EF 69 43), Łęki Górne (EF 78 13), Jaworze Dln. (EF 79 24), Słotowa (EF 79 31).

342. L. vulgaris L. - Wet meadows and fields: Podgrodzie (EF 69 44), Zwiernik (EF 78 34), Jaworze Dln. (EF 79 24).

343. Lythrum salicaria L. - Over banks of ditches: Parkosz (EF 69 43), Podgrodzie (EF 69 44), Jaworze Dln. (EF 79 24). 
344. Maianthemum bifolium (L.) F. W. Schmidt - In deciduous forests: Parkosz (EF 69 43), Podgrodzie (EF 69 44), Dobrków (EF 79 04), Gołęczyna (EF 79 14).

345. *Malva neglecta Wallr. - Archaeophyte. On ruderal habitats: Parkosz (EF 6943 ), Podgrodzie (EF 6944$)$.

346. ${ }^{\star}$ M. sylvestris L. - Archaeophyte. Roadsides: Podgrodzie (EF 69 44), Dobrków (EF 79 04), Jaworze Dln. (EF 79 24), Bielowy (EF 79 33).

347. *Matricaria maritima L. subsp. inodora (L.) Dostál - Archaeophyte. Fields and roadsides: Parkosz (EF 69 43), Mokrzec (EF 79 13), Bielowy (EF 79 33).

348. Matteucia struthiopteris (L.) Tod. - Lower montane zone species and partially protected. W olszynie nad potokiem: Jaworze Dln. (EF 79 24).

349. Medicago falcata L. - On balks: Parkosz (EF 69 43), Gołęczyna (EF 79 14).

350. M. lupulina L. - Meadows: Parkosz (EF 69 43), Pilzno (EF 79 12), Strzegocice (EF 7922 ).

351. ${ }^{\star}$ M. sativa L. - Hemiagriophyte. On meadows, balks and on roadsides: Parkosz (EF 69 43), Pilzno (EF 79 02), Jaworze Dln. (EF 79 24), Bielowy (EF 7933 ).

352. ${ }^{\star}$ M. $\times$ varia Martyn - Epoecophyte. On balk: Pilzno (EF 79 12).

353. Melampyrum arvense L. - On thermophilic meadows, fields: Parkosz (EF 69 43), Pilzno (EF 79 12).

354. M. nemorosum L. - In deciduous forests and thickets: Parkosz (EF 69 43), Podgrodzie (EF 69 44), Zwiernik (EF 78 34).

355. ${ }^{\star}$ Melandrium album (Mill.) Garcke - Archaeophyte. Meadows: Parkosz (EF 69 43), Łęki Grn. (EF 78 13), Pilzno (EF 79 02, EF 79 12), Gołęczyna (EF 79 14).

356. Melica nutans L. - In deciduous forests: Parkosz (EF 69 43), Zwiernik (EF 78 34).

357. Melilotus alba Medik. - On stones of rivers and on roadsides: Podgrodzie (EF 69 44), Dulczówka (EF 79 12), Jaworze Grn. (EF 79 34).

358. M. officinalis (L.) Pall. - On stones of rivers and on balks: Podgrodzie (EF 69 44), Jaworze Grn. (EF 79 34).

359. Mentha aquatica L. - On banks of ditches and ponds: Parkosz (EF 69 43), Dobrków (EF 79 04), Jaworze Dln.(EF 79 24).

360. M. arvensis L. - Fields and roadsides: Parkosz (EF 69 43), Pilzno (EF 79 02), Mokrzec (EF 79 13).

361. M. longifolia (L.) L. - On banks of streams, ditches and in wet forests: Parkosz (EF 69 43), Podgrodzie (EF 69 44), Łęki Grn.(EF 78 13), Gołęczyna (EF 79 14), Jaworze Dln. (EF 79 24).

362. Mercurialis perennis L. - In deciduous forests: Jaworze Dln. (EF 79 24), Zagórze (EF 79 43).

363. Milium effusum L. - In deciduous forests: Parkosz (EF 69 43), Podgrodzie (EF 69 44), Jaworze Dln.(EF 79 24).

364. Moehringia trinervia (L.) Clairv. - In forests and in thickets over the Wisłoka River: Podgrodzie (EF 69 44), Bielowy (EF 79 33). 
365. Moneses uniflora (L.) A. Gray - In pine forest: Jaworze Dln. (EF 79 24).

366. Monotropa hypopitys L. - In pine forest: Jaworze Dln. (EF 7924 ).

367. Mycelis muralis (L.) Dumort. - In forest: Parkosz (EF 69 43), Dobrków (EF 79 04).

368. ${ }^{\star}$ Myosotis arvensis (L.) Hill - Archaeophyte. On fields in cereal and root crops: Parkosz (EF 69 43), Łęki Grn. (EF 78 13), Pilzno (EF 79 12), Strzegocice (EF 79 22).

369. M. palustris (L.) L. emend. Rchb. - On wet meadows and on banks of ditches: Parkosz (EF 69 43), Zwiernik (EF 78 34), Dobrków (EF 79 04), Bielowy (EF 79 33).

370. M. sparsiflora Pohl. - In wet oak-hornbeam forest: Podgrodzie (EF 69 44).

371. M. stricta Link ex Roem. \& Schult. - Fields: Podgrodzie (EF 69 44), Bielowy (EF 79 33), Jaworze Dln. (EF 79 24).

372. M. sylvatica Ehrh. Ex Hoffm. - In wet oak-hornbeam forest: Pilzno (EF 79 12).

373. Myosoton aquaticum (L.) Moench - On stones of the Wisłoka River: Podgrodzie (EF 69 44)

374. Myriophyllum spicatum L. - In water reservoir: Jaworze Dln. (EF 79 24).

375. ${ }^{*}$ Nepeta cataria L. - Archaeophyte. Pastures: Parkosz (EF 69 43), Gołęczyna (EF 79 14).

376. Nuphar lutea (L.) Sibth. \& Sm. - In pond: Parkosz (EF 69 43).

377. Nymphaea candida C. Presl - Partially protected species. In water reservoir: Rędziny (EF 79 22).

378. Odontites serotina (Lam.) Rchb. - Pastures: Parkosz (EF 69 43), Jaworze Dln. (EF 79 24).

379. ${ }^{\star}$ O. verna (Bellardi) Dumort. - Archaeophyte. Fields: Parkosz (EF 69 43), Pilzno (EF 7902 ).

380. Oenanthe aquatica (L.) Poir. - On banks of ponds: Parkosz (EF 69 43), Podgrodzie (EF 69 44).

381. Oenothera biennis L. - On banks of rivers and roadsides: Podgrodzie (EF 69 44), Mokrzec (EF 79 13), Gołęczyna (EF 79 14).

382. Ononis arvensis L. - On balks and stones of the Wisłoka River: Podgrodzie (EF 69 44), Dobrków (EF 79 04), Słotowa (EF 7931 ).

383. ${ }^{\star}$ Onopordum acanthium L. - Archaeophyte. On ruderal habitats: Podgrodzie (EF 69 44).

384. Origanum vulgare L. - On balks: Podgrodzie (EF 69 44), Mokrzec (EF 79 13), Słotowa (EF 79 31).

385. Oxalis acetosella L. - In forest: Parkosz (EF 69 43), Podgrodzie (EF 69 44), Łęki Grn. (EF 78 13), Jaworze Dln. (EF 79 24).

386. ${ }^{\star}$ O. fontana Bunge - Epoecophyte. Fields: Parkosz (EF 69 43), Pilzno (EF 79 02, EF 79 12).

387. Padus avium Mill. - In moist forests and thickets: Parkosz (EF 69 43), Podgrodzie (EF 69 44), Łęki Grn. (EF 78 13).

388. ^Papaver argemone L. - Epoecophyte. Fields: Podgrodzie (EF 69 44), Gołęczyna (EF 79 14). 
389. *P. rhoeas L. - Archaeophyte. Fields: Parkosz (EF 69 43), Łęki Grn. (EF 78 13), Pilzno (EF 79 02), Bielowy (EF 7933 ).

390. Paris quadrifolia L. - In forests: Parkosz (EF 69 43), Podgrodzie (EF 69 44), Gołęczyna (EF 79 14).

391. *Parthenocissus inserta (A. Kern.) Fritsch - Holoagriophyte. Invasive species. In alluvial forests on rivers and streams: Gołęczyna (EF 79 14), Jaworze Dln.(EF 79 24).

392. ^Pastinaca sativa L. - Archaeophyte. Meadows: Podgrodzie (EF 6944 ).

393. Petasites albus (L.) Gaertn. - Higher montane zone species. In forests: Podgrodzie (EF 69 44), Jaworze Dln. (EF 79 24).

394. P. hybridus (L.) P. Gaertn., B. Mey. \& Scherb. - Over rivers and streams: Parkosz (EF 69 43), Podgrodzie (EF 69 44), Pilzno (EF 79 12).

395. Peucedanum oreoselinum (L.) Moench - On edges of pine forests: Gołęczyna (EF 79 14), Jaworze Dln. (EF 79 24).

396. Phalaris arundinacea L. - In reeds over banks of streams and ditches: Parkosz (EF 69 43), Podgrodzie (EF 69 44), Pilzno (EF 79 12).

397. Phegopteris connectilis (Michx.) Watt - In forests: Jaworze Dln. (EF 79 24).

398. Phleum pratense L. - Meadows: Parkosz (EF 69 43), Łęki Grn. (EF 78 13), Jaworze Dln. (EF 79 24), Bielowy (EF 79 33).

399. Phragmites australis (Cav.) Trin. ex Steud. - Over banks of drainage ditches: Jaworze Dln. (EF 79 24), Bielowy (EF 7933 ).

400. Picea abies (L.) H. Karst. - In forests, as an admixture: Zwiernik (EF 78 34), Gołęczyna (EF 79 14), Jaworze Dln. (EF 79 34).

401. Pimpinella maior (L.) Huds. - Meadow: Zwiernik.(EF 78 34).

402. P. saxifraga L. - On balks: Podgrodzie (EF 69 44), Strzegocice (EF 79 22).

403. Pinus sylvestris L. - In forests: Parkosz (EF 69 43), Słotowa (EF 79 31), Jaworze Dln. (EF 79 24).

404. Plantago intermedia Gilib. - Fields: Parkosz (EF 69 43), Pilzno (EF 79 02, EF 79 12).

405. P. lanceolata L. - Meadows, pastures and roadsides: Parkosz (EF 69 43), Podgrodzie (EF 69 44), Łęki Grn. (EF 78 13).

406. P. major L. - Meadows, pastures and roadsides: Parkosz (EF 69 43), Łęki Grn. (EF 78 13), Pilzno (EF 79 03), Jaworze Dln. (EF 79 24).

407. P. media L. - On dry meadows and balks: Parkosz (EF 69 34), Podgrodzie (EF 69 44), Dobrków (EF 79 04).

408. Platanthera bifolia (L.) Rich. - Partially protected species. In forests: Dobrków (EF 79 04), Bielowy (EF 7933 ).

409. Poa annua L. - Pastures and roadsides: Parkosz (EF 69 43), Podgrodzie (EF 69 44), Łęki Grn. (EF 78 13), Jaworze Dln. (EF 79 24).

410. P. compressa L. - On balks and on dry slopes of grasslands: Parkosz (EF 69 43), Zwiernik (EF 78 34), Jaworze Dln. (EF 79 24), Bielowy (EF 79 33). 
411. P. nemoralis L. - In forests: Parkosz (EF 69 43), Podgrodzie (EF 69 44), Jaworze Dln. (EF 79 24).

412. P. palustris L. - On alluvial of rivers and over banks of ponds: Parkosz (EF 69 43), Pilzno (EF 79 12).

413. P. pratensis L. - On meadows, balks and roadsides: Podgrodzie (EF 69 44), Łęki Grn. (EF 78 13), Strzegocice (EF 79 22), Jaworze Dln. (EF 79 24).

414. P. trivialis L. - Wet meadows and thickets: Parkosz (EF 69 43), Łęki Grn. (EF 78 13), Pilzno (EF 79 12), Strzegocice (EF 79 22).

415. Polygala comosa Schkuhr - On dry slopes of grasslands and in balks: Latoszyn (EF 69 44), Dobrków (EF 79 04).

416. P. oxyptera Rchb. - On dry and barren grasslands: Zagórze (EF 7933 ).

417. P. vulgaris L. - Dry meadows: Parkosz (EF 69 43), Dobrków (EF 7904 ).

418. Polygonatum multiflorum (L.) All. - In forests: Parkosz (EF 69 43), Podgrodzie (EF 69 44), Gołęczyna (EF 79 14).

419. Polygonum amphibium L. - On wet meadows and over banks of waters: Parkosz (EF 69 43), Pilzno (EF 79 12), Bielowy (EF 79 33).

420. P. aviculare L. - Fields and pastures: Parkosz (EF 69 43), Łęki Grn. (EF 78 13), Pilzno (EF 79 02), Bielowy (EF 79 33).

421. P. bistorta L. - Wet meadows: Parkosz (EF 6943 ).

422. P. hydropiper L. - Fields: Parkosz (EF 69 43), Zagórze (EF 79 33).

423. P. lapathifolium L. subsp. brittingeri (Opiz) Rech. F. - On ruderal habitats: Podgrodzie (EF 69 44), Pilzno (EF 79 12).

424. P. lapathifolium L. subsp. lapathifolium - Fields: Parkosz (EF 69 43), Podgrodzie (EF 69 44), Pilzno (EF 79 12).

425. P. lapathifolium L. subsp. pallidum (With.) Fr. - Fields, ruderal habitats: Parkosz (EF 69 43), Podgrodzie (EF 69 44), Pilzno (EF 79 02), Mokrzec (EF 79 13), Bielowy (EF 7933 ).

426. P. minus Huds. - On wet fields and over banks of ditches: Podgrodzie (EF 69 44), Jaworze Dln. (EF 79 24).

427. P. mite Schrank - Over ditches and on fields: Parkosz (EF 69 43), Podgrodzie (EF 6944 ).

428. P. persicaria L. - Ruderal habitats, fields: Parkosz (EF 69 43), Pilzno (EF 79 02), Mokrzec (EF 79 13).

429. Polypodium vulgare L. - In forests: Podgrodzie (EF 69 44), Jaworze Dln. (EF 79 24), Zagórze (EF 79 33).

430. Populus alba L. - In alluvial forests over the Wisłoka River: Parkosz (EF 69 43), Podgrodzie (EF 69 44), Pilzno (EF 79 12).

431. P. nigra L. - In alluvial forests over the Wisłoka River: Pilzno (EF 79 12), Strzegocice (EF 79 22).

432. P. tremula L. - In forests: Parkosz (EF 69 43), Łęki Grn. (EF 78 13), Słotowa (EF 79 31).

433. Potamogeton crispus L. - In still waters: Jaworze Dln. (EF 7924 ). 
434. P. natans L. - In bends of the Wisłoka River: Jaworze Dln. (EF 7924 ).

435. P. nodosus Poir. - In bends of the Wisłoka River: Jaworze Dln. (EF 79 24).

436. P. pusillus L. - In bends of the Wisłoka River: Jaworze Dln. (EF 79 24).

437. Potentilla anserina L. - On fields, pastures and roadsides: Podgrodzie (EF 69 44), Parkosz (EF 69 43), Pilzno (EF 79 03), Bielowy (EF 79 33).

438. P. argentea L. - Pastures: Podgrodzie (EF 69 44), Parkosz (EF 69 43), Słotowa (EF 79 11).

439. P. erecta (L.) Raeusch. - On edges of forests: Bielowy (EF 7933 ).

440. P. pusilla Host - Higher montane zone species. On stones of the Wisłoka River: Parkosz (EF 69 43).

441. P. reptans L. - Meadows and roadsides: Parkosz (EF 69 43), Pilzno (EF 79 02, EF 79 12), Jaworze Dln. (EF 79 24).

442. P. supina L. - Pastures: Parkosz (EF 69 43).

443. Primula elatior (L.) Hill - Partially protected species. In wet forests and meadows: Parkosz (EF 69 43), Jaworze Dln. (EF 79 24).

444. Prunella vulgaris L. - Meadows and pastures: Parkosz (EF 69 43), Dobrków (EF 7904 ).

445. Prunus spinosa L. - In thickets and on balks: Parkosz (EF 69 43), Podgrodzie (EF 6944 ), Jaworze Dln. (EF 79 24).

446. Pteridium aquilinum (L.) Kuhn - In mixed forests, in cutting places and balks: Parkosz (EF 69 43), Podgrodzie (EF 69 44), Gołęczyna (EF 79 14), Jaworze Dln. (EF 79 24).

447. Pulmonaria obscura Dumort. - In deciduous forests: Parkosz (EF 69 43), Podgrodzie (EF 69 44), Łęki Grn. (EF 78 13), Jaworze Dln. (EF 79 24).

448. Pyrola chlorantha Sw. - Partially protected species. In pine forest: Jaworze Dln. (EF 79 24).

449. P. minor L. - Partially protected species. In pine forest: Jaworze Dln. (EF 7924 ).

450. P. rotundifolia L. - Partially protected species. In pine forest: Jaworze Dln. (EF 7924 ).

451. Pyrus communis L. - In forests: Parkosz (EF 69 43), Podgrodzie (EF 69 44), Łęki Grn. (EF 78 13), Jaworze Dln. (EF 79 24), Bielowy (EF 79 33).

452. P. pyraster (L.) Burgsd. - On balks and in thickets: Parkosz (EF 69 43), Dobrków (EF 79 04), Mokrzec (EF 79 13).

453. Quercus petraea (Matt.) Liebl. - In forests: Parkosz (EF 69 43), Podgrodzie (EF 69 44), Gołęczyna (EF 79 14).

454. Q. robur L. - In forests: Łęki Grn. (EF 78 13), Gołęczyna (EF 79 14), Jaworze Dln. (EF 79 24).

455. ${ }^{\star}$ Q. rubra L. - Holoagriophyte. Invasive species. In forests: Dobrków (EF 79 04), Mokrzec (EF 79 13).

456. Ranunculus acris L. - Meadows: Parkosz (EF 69 43), Podgrodzie (EF 69 44), Łęki Grn. (EF 78 13), Zwiernik (EF 78 34), Pilzno (EF 79 03), Gołęczyna (EF 79 14), Jaworze Dln. (EF 79 24). 
457. ${ }^{\star} R$. arvensis L. - Archaeophyte. In cereal crops: Parkosz (EF 69 43), Podgrodzie (EF 69 44), Gołęczyna (EF 79 14).

458. $\boldsymbol{R}$. auricomus L. - Wet meadows: Parkosz (EF 69 43), Strzegocice (EF 79 22).

459. R. cassubicus L. - In forest: Parkosz (EF 69 43).

460. R. flammula L. - On banks of water reservoirs: Parkosz (EF 69 43).

461. R. lanuginosus L. - In forests: Parkosz (EF 69 43), Podgrodzie (EF 69 44).

462. R. polyanthemos L. - Dry meadows: Gołęczyna (EF 79 14), Jaworze Dln. (EF 79 24), Bielowy (EF 79 33).

463. R. repens L. - In meadows and ruderal habitats: Parkosz (EF 69 43), Łęki Grn. (EF 78 13), Pilzno (EF 79 12).

464. R. sardous Cranz - Pastures: Parkosz (EF 69 43), Dobrków (EF 7904 ).

465. ${ }^{\star}$ Raphanus raphanistrum L. - Archaeophyte. Fields: Parkosz (EF 69 43).

466. Reseda lutea L. - Roadsides: Podgrodzie (EF 69 44), Pilzno (EF 79 02), Gołęczyna (EF 79 14), Jaworze Dln. (EF 79 24).

467. ${ }^{\star}$ Reynotria japonica Houtt. - Holoagriophyte. Invasive species. In forests and on ruderal habitats: Gołęczyna (EF 79 14), Jaworze Dln. (EF 79 24).

468. Rhamnus cathartica L. - In thermophilic thickets on balks: Parkosz (EF 69 43), Podgrodzie (EF 69 44).

469. Rhinantus serotinus (Schönh.) Oborný - Meadows and fields: Parkosz (EF 69 43).

470. Ribes spicatum E. Robson - In forests: Jaworze Dln. (EF 79 24), Gołęczyna (EF 79 14).

471. ${ }^{\star}$ Robinia pseudoacacia L. - Holoagriophyte. Invasive species. In forests: Parkosz (EF 69 43), Mokrzec (EF 79 13).

472. Rorippa $\times$ armoracioides (Tausch) Fuss - On edge of thickets: Jaworze Dln. (EF 79 24).

473. R. austriaca (Cranz) Besser - Over the Wisłoka River: Pilzno (EF 79 12).

474. R. palustris (L.) Besser - Over the Wisłoka River: Pilzno (EF 79 12).

475. R. sylvestris (L.) Besser - Roadsides: Parkosz (EF 69 43), Podgrodzie (EF 69 44), Pilzno (EF 79 02), Gołęczyna (EF 79 14).

476. Rosa canina L. - In thickets, on balks and on edges of forests: Podgrodzie (EF 69 44), Zwiernik (EF 78 34), Jaworze Dln. (EF 79 24), Bielowy (EF 79 33), Jaworze Grn. (EF 79 34).

477. R. rubiginosa L. - In thickets over the Wisłoka River: Jaworze Grn. (EF 79 34).

478. Rubus bifrons Vest - On edge of thickets: Podgrodzie (EF 69 44).

479. R. caesius L. - Thickets: Parkosz (EF 69 43), Podgrodzie (EF 69 44), Łęki Grn. (EF 78 13), Słotowa (EF 79 31).

480. R. hirtus Waldst. \& Kit. - Forests and thickets: Pilzno (EF 79 12), Gołęczyna (EF 79 14), Jaworze Dln. (EF 79 24).

481. R. idaeus L. - Forests and thickets: Podgrodzie (EF 69 44), Łęki Grn. (EF 78 13), Gołęczyna (EF 79 14), Jaworze Dln. (EF 79 24), Bielowy (EF 7933 ).

482. R. montanus Lib. ex Lej. - Thickets: Parkosz (EF 69 43), Jaworze Dln. (EF 79 24). 
483. R. plicatus Weihe \& Nees - In thickets over the Wisłoka River: Podgrodzie (EF 6944 ), Jaworze Dln. (EF 79 24).

484. ${ }^{\star}$ Rudbeckia laciniata L. - Holoagriophyte. Invasive species. In alluvial forests: Łęki Grn. (EF 78 13), Dulczówka (EF 79 12).

485. Rumex acetosa L. - Meadows: Podgrodzie (EF 69 44), Słotowa (EF 79 31), Jaworze Dln. (EF 7924$)$.

486. R. acetosella L. - Fields: Podgrodzie (EF 69 44), Łęki Grn. (EF 78 13), Jaworze Dln. (EF 79 24), Bielowy (EF 79 33).

487. R. aquaticus L. - On bank of ditch: Strzegocice (EF 79 22).

488. R. conglomeratus Murray - In ditches and on wet meadows: Parkosz (EF 69 43), Podgrodzie (EF 69 44), Dobrków (EF 79 04), Jaworze Dln. (EF 79 24).

489. R. crispus L. - Meadows: Łęki Grn. (EF 78 13), Dobrków (EF 7904 ).

490. R. obtusifolius L. - Meadows: Parkosz (EF 69 43), Podgrodzie (EF 69 44), Pilzno (EF 79 12), Gołęczyna (EF 79 14), Jaworze Dln. (EF 79 24), Słotowa (EF 7931 ).

491. R. sanguineus L. - Wet forests: Podgrodzie (EF 69 44), Jaworze Dln. (EF 79 24).

492. R. thyrsiflorus Fingerh. - On meadows and on roadside slopes: Parkosz (EF 69 43), Pilzno (EF 79 12).

493. Sagina procumbens L. - Wet meadows: Jaworze Dln. (EF 79 24).

494. Salix alba L. - In alluvial forests over the Wisłoka River: Podgrodzie (EF 69 44), Łęki Grn. (EF 78 13), Pilzno (EF 79 12), Słotowa (EF 79 31).

495. S. caprea L. - Thickets: Podgrodzie (EF 69 44), Strzegocice (EF 7922 ).

496. S. cinerea L. - In riparian forest over stream: Strzegocice (EF 7922 ).

497. S. fragilis L. - In riparian forests over streams: Łęki Grn. (EF 78 13), Parkosz (EF 69 43).

498. S. purpurea L. - In riparian forests over streams: Podgrodzie (EF 69 44), Łęki Grn. (EF 78 13), Jaworze Dln. (EF 79 24).

499. S. triandra L. - In alluvial forests over the Wisłoka River: Parkosz (EF 69 43), Pilzno (EF 79 12), Strzegocice (EF 79 22).

500. S. viminalis L. - In riparian forests over rivers: Łęki Grn. (EF 78 13), Parkosz (EF 69 43), Jaworze Dln. (EF 79 24).

501. Salvia glutinosa L. - Higher montane zone species. In forests: Podgrodzie (EF 69 34), Parkosz (EF 69 43), Gołęczyna (EF 79 14).

502. Sambucus ebulus L. - On balks: Dobrków (EF 79 04), Bielowy (EF 7933 ).

503. S. nigra L. - Forests and thickets: Parkosz (EF 69 43), Podgrodzie (EF 69 44), Łęki Grn. (EF 78 13), Pilzno (EF 79 12), Gołęczyna (EF 79 14), Jaworze Dln. (EF 79 24), Bielowy (EF 7933 ), Jaworze Grn. (EF 7934 ).

504. S. racemosa L. - Higher montane zone species. In forests: Podgrodzie (EF 69 44), Jaworze Dln. (EF 79 24).

505. Sanguisorba minor Scop. - On dry slope: Jaworze Dln. (EF 79 24). 
506. Sanicula europaea L. - In forests: Podgrodzie (EF 69 44), Zwiernik (EF 78 34), Jaworze Grn. (EF 79 34).

507. Saponaria officinalis L. - Roadsides: Podgrodzie (EF 69 44), Pilzno (EF 79 12), Gołęczyna (EF 79 14).

508. Sarothamnus scoparius (L.) W. D. J. Koch - On edge of forest: Jaworze Dln. (EF 79 24).

509. Scilla bifolia L. - All-mountain species, partially protected. In forests: Parkosz (EF 69 43), Podgrodzie (EF 69 44).

510. Scirpus sylvaticus L. - On wet meadows and over banks of ditches: Podgrodzie (EF 69 44), Dobrków (EF 79 04), Bielowy (EF 79 33).

511. ^Scleranthus annuus L. - Archaeophyte. Fields: Parkosz (EF 69 43), Podgrodzie (EF 69 44), Mokrzec (EF 79 13), Gołęczyna (EF 79 14), Zagórze (EF 7933 ).

512. S. perennis L. - On sandy dunes and fallow lands: Podgrodzie (EF 69 44), Gołęczyna (EF 79 14), Jaworze Dln. (EF 79 24).

513. S. polycarpos L. - On sandy fallow land: Gołęczyna (EF 79 14).

514. Scrophularia nodosa L. - Thickets: Parkosz (EF 69 43).

515. S. umbrosa Dumort. - Over banks of pond: Jaworze Dln. (EF 7924 ).

516. Sedum acre L. - On stones of the Wisłoka River: Parkosz (EF 69 43), Podgrodzie (EF 69 44), Gołęczyna (EF 79 14), Jaworze Dln. (EF 79 24).

517. S. maximum (L.) Hoffm. - On roadside slopes and balks: Parkosz (EF 69 43), Podgrodzie (EF 69 44), Zwiernik (EF 78 34), Jaworze Dln. (EF 79 24).

518. S. sexangulare L. - On stones of the Wisłoka River: Podgrodzie (EF 69 44), Parkosz (EF 69 43)

519. Selinum carvifolia (L.) L. - On edge of forest: Strzegocice (EF 79 22).

520. Senecio barbaraeifolius (Krock.) Wimm. \& Grab. - Wet meadows: Jaworze Dln. (EF 79 24), Dęborzyn (EF 79 44).

521. S. fluviatilis Wallr. - In thickets on the Wisłoka River: Parkosz (EF 69 43), Podgrodzie (EF 69 44), Pilzno (EF 79 12), Mokrzec (EF 79 13).

522. S. jacobaea L. - Roadsides: Parkosz (EF 69 43), Podgrodzie (EF 69 44), Dobrków (EF 79 04).

523. S. ovatus (P. Gaertn., B. Mey. \& Schreb.) Willd. - Higher montane zone species. In forests and on cutting places: Zwiernik (EF 78 34), Jaworze Grn. (EF 79 34).

524. S. sylvaticus L. - On cutting places: Jaworze Dln. (EF 7924 ).

525. ${ }^{\ngtr}$ S. vernalis Waldst. \& Kit. - Epoecophyte. Roadside: Jaworze Dln. (EF 79 24).

526. S. viscosus L. - On stones of the Wisłoka River: Podgrodzie (EF 69 34).

527. ${ }^{\star}$ S. vulgaris L. - Archaeophyte. On ruderal habitats: Pilzno (EF 79 12).

528. Seseli annuum L. - In pine forests: Jaworze Dln. (EF 79 24).

529. ${ }^{\star}$ Setaria pumila (Poir.) Roem. \& Schult. - Archaeophyte. Fields: Parkosz (EF 69 43) Pilzno (EF 79 02). 
530. ${ }^{\star}$ S. viridis (L.) P. Beauv. - Archaeophyte. Fields: Parkosz (EF 69 43). Podgrodzie (EF 69 44), Dobrków (EF 79 04), Pilzno (EF 79 12), Jaworze Dln. (EF 79 24).

531. ^Sherardia arvensis L. - Archaeophyte. Fields: Parkosz (EF 69 43), Pilzno (EF 79 02), Dobrków (EF 79 04), Bielowy (EF 7933 ).

532. Silene nutans L. subsp. nutans - Dry meadow: Jaworze Dln. (EF 7924 ).

533. S. vulgaris (Moench) Garcke - Meadows and roadsides: Podgrodzie (EF 69 44), Zwiernik (EF 78 34), Gołęczyna (EF 79 14), Jaworze Dln. (EF 79 24).

534. `Sinapis arvensis L. - Archaeophyte. Fields: Parkosz (EF 69 43), Pilzno (EF 7902 , EF 79 12).

535. ${ }^{\star}$ Sisymbrium officinale (L.) Scop. - Archaeophyte. In fields and ruderal habitats: Parkosz (EF 69 43), Zwiernik (EF 78 34), Pilzno (EF 79 12).

536. Solanum dulcamara L. - Wet forests: Dobrków (EF 79 04), Jaworze Dln. (EF 79 24).

537. ${ }^{\star S}$. nigrum L. emend. Mill. - Archaeophyte. Roadsides: Parkosz (EF 69 43), Pilzno (EF 79 12), Mokrzec (EF 79 13).

538. ${ }^{\star}$ Solidago gigantea Aiton - Holoagriophyte. Invasive species. In alluvial forests over rivers and on roadsides: Podgrodzie (EF 69 44), Łęki Grn. (EF 78 13), Pilzno (EF 79 12), Mokrzec (EF 79 13), Gołęczyna (EF 79 14), Jaworze Dln. (EF 79 24).

539. S. virgaurea L. - In mixed forests: Jaworze Dln. (EF 79 24), Bielowy (EF 79 33).

540. Sonchus arvensis L. - Fields and roadsides: Parkosz (EF 69 43), Podgrodzie (EF 69 44), Pilzno (EF 79 02), Dobrków (EF 79 04), Słotowa (EF 7931 ).

541. ${ }^{\star}$ S. asper (L.) Hill - Archaeophyte. In fields of root crops and in ruderal habitats: Parkosz (EF 69 43), Dobrków (EF 79 04).

542. ${ }^{\star}$ S. oleraceus L. - Archaeophyte. In fields of root crops: Dobrków (EF 79 04).

543. Sorbus aucuparia L. emend. Hedl. - Forests and thickets: Parkosz (EF 69 43), Gołęczyna (EF 79 14), Jaworze Dln. (EF 79 24).

544. `Spergula arvensis L. - Archaeophyte. Fields: Parkosz (EF 69 43), Podgrodzie (EF 69 44), Słotowa (EF 79 31), Jaworze Dln. (EF 79 24).

545. S. morisonii Boreau - On sand dune: Jaworze Dln. (EF 79 24).

546. Spergularia rubra (L.) J. Presl \& C. Presl - On sandy fallow lands: Gołęczyna (EF 79 14), Jaworze Dln. (EF 79 24).

547. Spirodela polyrhiza (L.) Schleid. - In pond: Mokrzec (EF 79 14).

548. Stachys palustris L. - On bank of pond: Parkosz (EF 69 43).

549. S. sylvatica L. - In forests: Parkosz (EF 69 43), Podgrodzie (EF 69 44), Jaworze Dln. (EF 79 24), Bielowy (EF 79 33).

550. Staphyllea pinnata L. - Protected species. In oak-hornbeam forest: Podgrodzie (EF 69 44). 551. Stellaria graminea L. - Meadows: Parkosz (EF 69 43), Podgrodzie (EF 69 44), Łęki Grn. (EF 78 13), Dobrków (EF 79 04), Pilzno (EF 79 12).

552. S. holostea L. - In oak-hornbeam forests: Parkosz (EF 69 43), Podgrodzie (EF 69 44), Dobrków (EF 79 04), Bielowy (EF 79 33). 
553. S. media (L.) Vill. - In forests and thickets and in fields and ruderal habitats: Parkosz (EF 69 43), Podgrodzie (EF 69 44), Łęki Grn. (EF 78 13), Pilzno (EF 79 02), Słotowa (EF 79 31).

554. S. nemorum L. - In forests: Parkosz (EF 69 43), Podgrodzie (EF 69 44), Jaworze Dln. (EF 79 24).

555. S. uliginosa Murray - Wet meadows: Zwiernik (EF 78 34).

556. Symphytum officinale L. - On moist meadows, banks of ditches and on roadsides: Parkosz (EF 69 43), Podgrodzie (EF 69 44), Pilzno (EF 79 02).

557. S. tuberosum L. - In oak-hornbeam forests and alluvial forests: Parkosz (EF 69 43), Podgrodzie (EF 69 44), Jaworze Dln. (EF 79 24), Słotowa (EF 79 31).

558. ${ }^{\star}$ Syringa vulgaris L. - Holoagriophyte. In thickets: Parkosz (EF 6943 ).

559. ${ }^{\star}$ Tanacetum parthenium (L.) Sch. Bip. - Epoecophyte. Roadsides: Dobrków (EF 79 04), Mokrzec (EF 79 13).

560. T. vulgare L. - On pastures, roadsides and in ruderal habitats: Parkosz (EF 69 43), Podgrodzie (EF 69 44), Łęki Grn. (EF 78 13), Pilzno (EF 79 03), Strzegocice (EF 79 22).

561. Taraxacum officinale F. H. Wigg. - Meadows, fields and roadsides: Podgrodzie (EF 69 44), Łęki Grn. (EF 78 13), Pilzno (EF 79 02, EF 79 03), Dobrków (EF 79 04), Gołęczyna (EF 79 14).

562. Teesdalea nudicaulis (L.) R. Br. - On sandy fields: Parkosz (EF 69 43), Gołęczyna (EF 79 14), Jaworze Dln. (EF 79 24).

563. ${ }^{\star}$ Telekia speciosa (Schreb.) Baumg. - Holoagriophyte. In alder forest above the stream: Jaworze Dln. (EF 79 24).

564. ${ }^{\star}$ Thlaspi arvense L. - Archaeophyte. In fields and in ruderal habitats: Parkosz (EF 69 43), Pilzno (EF 79 03, EF 79 12).

565. Thymus pulegioides L. - On dry meadows and balks: Parkosz (EF 69 43), Pilzno (EF 79 03), Dobrków (EF 79 04).

566. T. serpyllum L. emend. Fr. - In pine forests: Gołęczyna (EF 79 14), Jaworze Dln. (EF 79 24).

567. Torilis japonica (Houtt.) DC. - In thickets over the Wisłoka River: Podgrodzie (EF 69 44), Mokrzec (EF 79 13).

568. Tragopogon orientalis L. - Meadows: Jaworze Grn. (EF 79 34).

569. Trientalis europaea L. - In pine forests: Gołęczyna (EF 79 14), Jaworze Dln. (EF 79 24).

570. Trifolium arvense L. - Roadsides: Podgrodzie (EF 69 44), Jaworze Dln. (EF 79 24).

571. T. aureum Pollich - On balks: Parkosz (EF 69 43), Dobrków (EF 7904 ).

572. T. campestre Schreb. - In grasslands on dry slopes and in meadows: Podgrodzie (EF 69 44), Łęki Grn. (EF 78 13), Jaworze Dln. (EF 79 24).

573. T. dubium Sibth. - Meadows: Podgrodzie (EF 69 44), Zwiernik (EF 78 34), Pilzno (EF 79 12).

574. T. fragiferum L. - On wet meadows and pastures: Podgrodzie (EF 69 44), Dobrków (EF 79 04). 
575. T. hybridum L. subsp. hybridum - Wet meadows. Parkosz (EF 69 43), Dobrków (EF 79 04), Słotowa (EF 7931 ).

576. T. medium L. - On balks and dry meadows: Parkosz (EF 69 43), Dobrków (EF 79 04).

577. T. pratense L. - On stones of the Wisłoka River and on meadows: Podgrodzie (EF 69 44), Łęki Grn. (EF 78 13), Dulczówka (EF 79 12).

578. T. repens L. - Meadows: Parkosz (EF 69 43), Łęki Grn. (EF 78 13), Gołęczyna (EF 79 14), Jaworze Dln. (EF 79 24).

579. Trisetum flavescens (L.) P. Beauv. - Meadows: Łęki Grn. (EF 78 13), Jaworze Dln. (EF $7924)$.

580. Tussilago farfara L. - In fields, balks and ruderal habitats: Parkosz (EF 69 43), Podgrodzie (EF 69 44), Pilzno (EF 79 02, EF 79 03), Jaworze Dln. (EF 79 24), Bielowy (EF 7933 ).

581. Typha latifolia L. - In water reservoirs: Parkosz (EF 69 43), Dobrków (EF 79 04), Dulczówka (EF 79 12), Mokrzec (EF 79 13), Jaworze Dln. (EF 79 24).

582. Ulmus glabra Huds. - Oak-hornbeam forests: Podgrodzie (EF 69 44), Jaworze Dln. (EF 79 24).

583. U. laevis Pall. - Oak-hornbeam forests: Parkosz (EF 69 43), Słotowa (EF 79 31).

584. Urtica dioica L. - In moist deciduous forests and ruderal habitats: Parkosz (EF 69 43), Łęki Grn. (EF 78 13), Pilzno (EF 79 03), Jaworze Dln. (EF 79 24).

585. * U. urens L. - Archaeophyte. On ruderal habitats: Parkosz (EF 69 43), Mokrzec (EF 79 13), Gołęczyna (EF 79 14).

586. Vaccinium myrtillus L. - In mixed forests: Podgrodzie (EF 69 44), Zwiernik (EF 78 34), Gołęczyna (EF 79 14).

587. V. vitis-idaea L. - In mixed forests: Podgrodzie (EF 69 44), Dobrków (EF 79 04), Gołęczyna (EF 79 14).

588. Valeriana simplicifolia Kabath - Wet meadows: Parkosz (EF 69 43), Dobrków (EF 79 04), Bielowy (EF 79 33).

589. ${ }^{\star}$ Valerianella dentata (L.) Pollich - Archaeophyte. Fields: Pilzno (EF 79 02), Dobrków (EF 7904$)$.

590. ${ }^{\star}$ V. rimosa Bastard - Archaeophyte. Fields: Parkosz (EF 69 43).

591. Verbascum densiflorum Bertol. - On gravels of the Wisłoka River: Parkosz (EF 69 43), Jaworze Dln. (EF 79 24).

592. V. phlomoides L. - Roadsides: Podgrodzie (EF 69 44).

593. V. thapsus L. - On dry slope: Parkosz (EF 69 43).

594. *Verbena officinalis L. - Archaeophyte. On ruderal habitats: Mokrzec (EF 79 13).

595. Veronica anagallis-aquatica L. - On banks of streams and drainage ditches: Podgrodzie (EF 69 44), Dobrków (EF 79 04).

596. ${ }^{\star}$ V. arvensis L. - Archaeophyte. Fields: Parkosz (EF 69 43), Podgrodzie (EF 69 44), Zwiernik (EF 78 34), Strzegocice (EF 79 22), Jaworze Dln. (EF 79 24). 
597. V. beccabunga L. - On banks of streams: Podgrodzie (EF 69 44), Jaworze Dln. (EF 79 24), Bielowy (EF 79 33).

598. V. chamaedrys L. - Roadsides: Parkosz (EF 69 43), Podgrodzie (EF 69 44), Łęki Grn. (EF 78 13), Pilzno (EF 79 03), Gołęczyna (EF 79 14).

599. V. hederifolia L. - Fields: Parkosz (EF 69 43), Podgrodzie (EF 69 44), Gołęczyna (EF 79 14).

600. V. montana L. - Oak-hornbeam forests: Podgrodzie (EF 69 44), Zwiernik (EF 78 34), Jaworze Dln. (EF 79 24).

601. V. officinalis L. - In forests, on their outskirts and in fallow areas: Zwiernik (EF 78 34), Gołęczyna (EF 79 14), Jaworze Dln. (EF 79 24), Jaworze Grn. (EF 79 34).

602. * V. persica Poir. - Epoecophyte. Fields: Parkosz (EF 69 43), Podgrodzie (EF 69 44), Łęki Grn. (EF 78 13), Pilzno (EF 79 02), Strzegocice (EF 79 22).

603. * V. polita Fr. - Archaeophyte. Fields: Parkosz (EF 69 43), Podgrodzie (EF 69 44), Dobrków (EF 79 04).

604. V. serpyllifolia L. - Fields: Podgrodzie (EF 69 44), Strzegocice (EF 79 22).

605. V. spicata L. - On sand dune: Podgrodzie (EF 69 44).

606. ${ }^{*}$ V. triphyllos L. - Archaeophyte. Fields: Podgrodzie (EF 69 44), Gołęczyna (EF 79 14).

607. Viburnum opulus L. - In alluvial forests: Podgrodzie (EF 69 44), Parkosz (EF 69 43), Łęki Grn. (EF 78 13), Dobrków (EF 79 04).

608. ${ }^{*}$ Vicia angustifolia L. - Archaeophyte. In field communities: Parkosz (EF 69 43), Podgrodzie EF 69 44), Zwiernik (EF 78 34), Pilzno (EF 79 12), Gołęczyna (EF 79 14).

609. V. cracca L. - Meadows, fields and roadsides: Parkosz (EF 69 43), Podgrodzie (EF 69 44), Łęki Grn. (EF 78 13), Pilzno (EF 79 12), Gołęczyna (EF 79 14), Jaworze Dln. (EF 79 24).

610. ${ }^{\star}$ V. dasycarpa Ten. - Epecophyte. Fields: Parkosz (EF 69 43), Pilzno (EF 79 02), Dobrków (EF 79 04), Bielowy (EF 7933 ).

611. ${ }^{\star}$ V. hirsuta (L.) Gray - Archaeophyte. On cereal fields. Parkosz (EF 69 43), Podgrodzie (EF 69 44), Zwiernik (EF 78 34), Pilzno (EF 79 12), Gołęczyna (EF 79 14).

612. V. sepium L. - Thickets: Parkosz (EF 69 43), Zwiernik (EF 78 34), Pilzno (EF 79 12).

613. ${ }^{\star} V$. tetrasperma (L.) Schreb. - Archaeophyte. On cereal fields: Łęki Grn. (EF 78 13), Gołęczyna (EF 79 14), Słotowa (EF 79 31).

614. ${ }^{\star}$ V. villosa Roth - Archaeophyte. On cereal fields: Parkosz (EF 69 43), Mokrzec (EF 79 13).

615. Vinca minor L. - Oak-hornbeam forests over the Wisłoka River: Podgrodzie (EF 6944 ).

616. ${ }^{*}$ Viola arvensis Murray - Archaeophyte. Fields: Parkosz (EF 69 43), Podgrodzie (EF 69 44), Pilzno (EF 79 02), Gołęczyna (EF 79 14), Jaworze Dln. (EF 79 24).

617. V. canina L. - On edges of forests: Gołęczyna (EF 79 14), Jaworze Dln. (EF 79 24), Bielowy (EF 79 33).

618. V. hirta L. - In thickets over the Wisłoka River: Podgrodzie (EF 69 44). 
619. V. odorata L. - In riparian forests and wet oak-hornbeam forests: Parkosz (EF 69 43), Podgrodzie (EF 69 44), Łęki Grn. (EF 78 13).

620. V. reichenbachiana Jord. ex Boreau - Oak-hornbeam forests: Parkosz (EF 69 43), Podgrodzie (EF 69 44), Jaworze Dln. (EF 79 24).

621. V. riviniana Rchb. - Oak-hornbeam forest: Parkosz (EF 69 43).

622. V. rupestris F. W. Schmidt - On sandy fallow area: Jaworze Dln. (EF 7924 ).

623. V. tricolor L. - In cereals on sandy fields: Gołęczyna (EF 79 14), Bielowy (EF 7933 ).

624. ${ }^{\star}$ Xanthium albinum (Widder) H. Scholz - Hemiagriophyte. On the Wisłoka River alluvials: Parkosz (EF 69 43).

625. ${ }^{\star} X$. strumarium L. - Epoecophyte. On ruderal habitats: Parkosz (EF 69 43), Podgrodzie (EF 69 44), Mokrzec (EF 79 13), Jaworze Dln. (EF 79 24).

Summary

The list of vascular plants presented above has 625 taxa, of which 514 are native and the remaining 111 are alien taxa. Within them 70 are archaeophytes, 21 are epoecophytes, 6 are classified as hemiagriophytes, and 14 are holoagriophytes. (Appendix $1 \mathrm{E}-\mathrm{G})$. Thirteen alien species have the status of invasive plants. Among native plants, 19 mountain species were found: 4 lower montane zone species, 12 higher montane zone species and 3 all-mountainous. Eleven protected species have been reported on study area, among others orchids: Cephalanthera longifolia, Epipactis purpurata (complete protection), or Dactylorhiza majalis (partial protection).

\section{Conflict of interest}

The author declares no conflict of interest related to this article.

\section{References}

Kondracki, J. (1978). Geografia fizyczna Polski. Warszawa: Wydawnictwo PWN. [In Polish]

Kornaś, J., Medwecka-Kornaś, A., Towpasz, K. (1997). Rośliny naczyniowe Pogórza Ciężkowickiego. Zeszyty Naukowe Uniwersytetu Jagiellońskiego, Prace Botaniczne, 28, 1-170. [In Polish]

Mirek, Z., Piękoś-Mirkowa, H., Zając, A., Zając, M. (2002). Flowering plants and Pteridophytes of Poland - a checklist. In: Z. Mirek (ed.), Biodiversity of Poland, 1-442. 1. Kraków: W. Szafer Institute of Botany, Polish Academy of Sciences.

Oklejewicz, K., Jóźwiak, A., Skrzypek, B., Wolak, N. (2004). Uzupełnienie do flory Pogórza Strzyżowskiego (SE Polska). Fragmenta Floristica et Geobotanica Polonica, 11, 199-202. [In Polish]

Pawłowski, B. (1925). Geobotaniczne stosunki Sądeczyzny. Prace Monograficzne Komisji Fizjograficznej. Kraków: Wyd. Polska Akademia Umiejętności. [In Polish]

Pawłowski, B. (1972). Szata roślinna gór polskich. In: W. Szafer, K. Zarzycki (eds.), Szata roślinna Polski, 1-342. 2. Warsaw: Polish Scientific Publishers PWN. [In Polish]

Regulation of the Minister of the Environment of October 9, 2014 on the protection of plant species (Rozporządzenie Ministra Środowiska z dnia 9 października 2014 r. w sprawie ochrony gatunkowej roślin) Dz.U. z 2014 r. poz. 1409. [In Polish] 
Tokarska-Guzik, B., Dajdok, Z., Zając, M., Zając, A., Urbisz A., Danielewicz, W., Hołdyński, C. (2012). Rośliny obcego pochodzenia ze szczególnym uwzględnieniem gatunków inwazyjnych. Warszawa: Generalna Dyrekcja Ochrony Środowiska. [In Polish]

Towpasz, K. (1987). Rośliny naczyniowe Pogórza Strzyżowskiego. Zeszyty Naukowe Uniwersytetu Jagiellońskiego, Prace Botaniczne, 16, 1-160. [In Polish]

Towpasz, K. (1990). Charakterystyka geobotaniczna Pogórza Strzyżowskiego. Zeszyty Naukowe Uniwersytetu Jagiellońskiego, Rozprawy Habilitacyjne, 178, 1-242. [In Polish]

Towpasz, K. (2013). Uzupełnienia do flory roślin naczyniowych Pogórza Strzyżowskiego (Karpaty Zachodnie). Część II. Fragmenta Floristica et Geobotanica Polonica, 20(1), 41-50. [In Polish]

Towpasz, K. (2018). Środowisko naturalne okolic Pilzna. In: B. Stanaszek (ed.), Pilzno, monografia miasta do 1945 roku, 11-30. Pilzno: Towarzystwo Przyjaciół Pilzna i Ziemi Pilźnieńskiej [In Polish]

Wójcik, T. (2011). Notatki florystyczne ze Strzyżowa i okolic (Pogórze Strzyżowskie). Fragmenta Floristica et Geobotanica Polonica, 18(1), 83-90. [In Polish]

Zając, A. (1978). Założenia metodyczne „Atlasu rozmieszczenia roślin naczyniowych w Polsce”. Wiadomości Botaniczne, 22(3), 145-155. [In Polish]

Zając, A., Zając, M. (2001). Distribution Atlas of Vascular Plants in Poland. Nakładem Pracowni Chorologii Komputerowej Instytutu Botaniki UJ, Kraków s. XII + 716. [In Polish/English]

Zając, A., Zając, M. (2015). Distribution of kenophytes in the Polish Carpatians and their foreland. Kraków: Nakładem Instytutu Botaniki Uniwersytetu Jagiellońskiego. [In Polish/English] 


\section{Appendix 1}

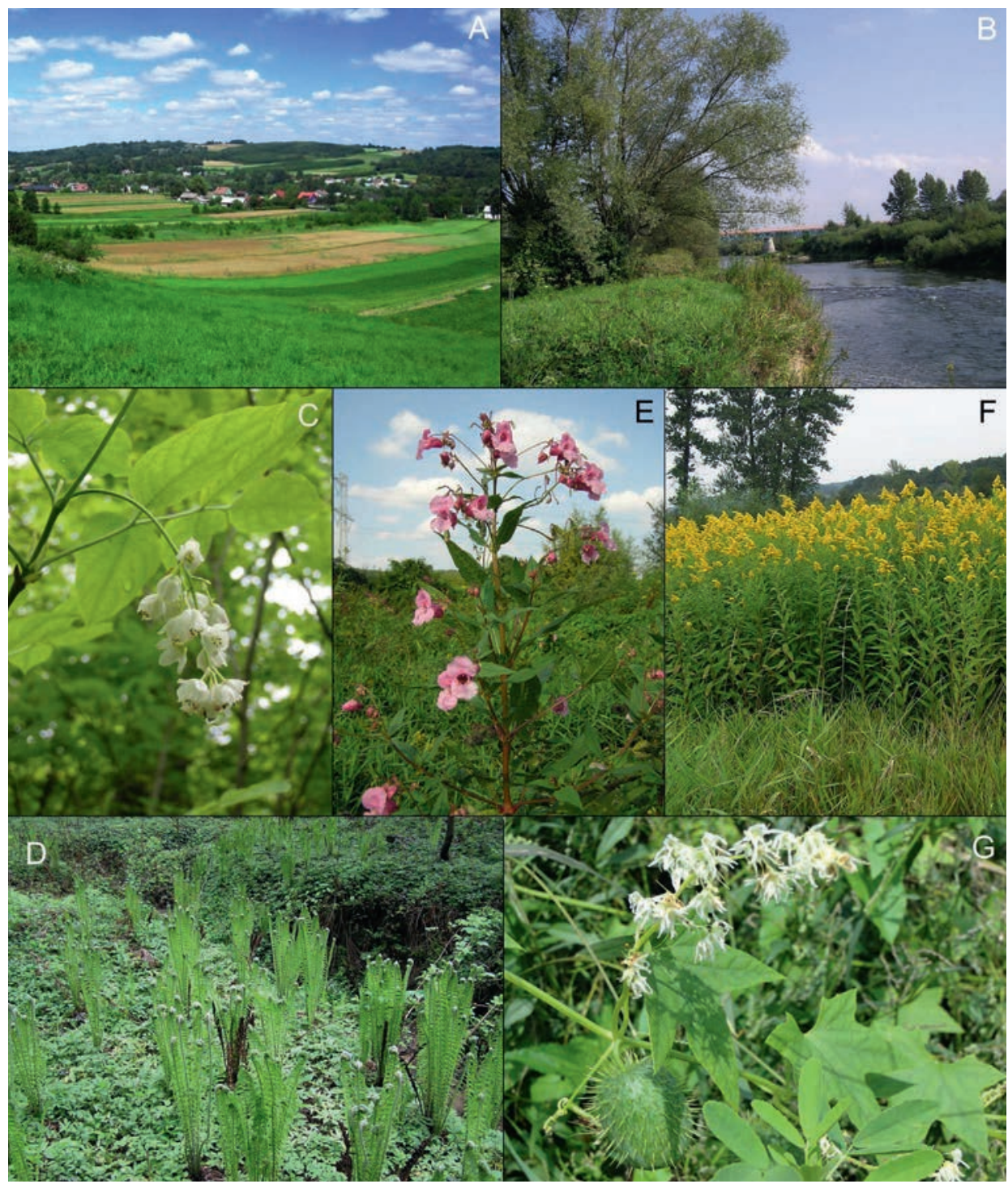

Dulczówka near Pilzno, agricultural landscape - A; alluvial forest over the Wisłoka River - B; protected species: Staphyllea pinnata L. - C, Matteucia struthiopteris (L.) Tod. - D; invasive holoagriophytes: Impatiens glandulifera Royle - E, Solidago gigantea Aiton - F, Echinocystis lobata (F. Michx.) Torr. \& A. Gray - G, (Photo. K. Towpasz) 
The paper presents the occurrence of vascular plant species in the southern part of the Pilzno commune based on monographic studies from the area of Ciężkowice and Strzyżów Foothills (Western Carpathians). The study contains a list of plant species, both native and of alien origin. For each species its habitat and sites in the ATPOL network were given.

Key words: vascular plants, distribution, Pilzno commune, Ciężkowice Foothills, Strzyżów Foothills

Received: [2019.09.05]

Accepted: [2019.11.22]

\section{Rośliny naczyniowe okolic Pilzna (Południowo-Wschodnia Polska)}

Streszczenie

W pracy przedstawiono występowanie gatunków roślin naczyniowych na obszarze południowej części gminy Pilzno w oparciu o opracowania monograficzne z terenu Pogórzy Ciężkowickiego i Strzyżowskiego (Karpaty Zachodnie). Artykuł zawiera wykaz roślin, zarówno rodzimych, jak i obcego pochodzenia. Dla każdego gatunku podano jego siedlisko i lokalizację w sieci ATPOL.

Słowa kluczowe: rośliny naczyniowe, rozmieszczenie, gmina Pilzno, Pogórze Ciężkowickie, Pogórze Strzyżowskie

\section{Information about author}

\section{Krystyna Towpasz}

She is retired professor at the Institute ob Botany of the Jagiellonian University in Kraków. She is interested in geobotany, plant ecology and plant protection. 